\title{
Local Stability in 3D Discrete Dynamical Systems: Application to a Ricker Competition Model
}

\author{
Rafael Luís $^{1,2}$ and Elias Rodrigues ${ }^{1}$ \\ ${ }^{1}$ University of Madeira, Funchal, Madeira, Portugal \\ ${ }^{2}$ Center for Mathematical Analysis, Geometry, and Dynamical Systems, University of Lisbon, Lisbon, Portugal \\ Correspondence should be addressed to Rafael Luís; rafael.luis.madeira@gmail.com
}

Received 31 January 2017; Revised 3 May 2017; Accepted 22 May 2017; Published 6 July 2017

Academic Editor: Mustafa Kulenović

Copyright (c) 2017 Rafael Luís and Elias Rodrigues. This is an open access article distributed under the Creative Commons Attribution License, which permits unrestricted use, distribution, and reproduction in any medium, provided the original work is properly cited.

\begin{abstract}
A survey on the conditions of local stability of fixed points of three-dimensional discrete dynamical systems or difference equations is provided. In particular, the techniques for studying the stability of nonhyperbolic fixed points via the centre manifold theorem are presented. A nonlinear model in population dynamics is studied, namely, the Ricker competition model of three species. In addition, a conjecture about the global stability of the nontrivial fixed points of the Ricker competition model is presented.
\end{abstract}

\section{Introduction}

In the study of a dynamical system, specially of nonlinear models, one of the objectives is to determine the stability conditions of equilibrium points or fixed points.

According to Osipenko [1], in his excellent paper titled "Center Manifolds", Lyapunov [2], in his dissertation "General Problem on Stability of Motion," was perhaps one of the pioneers in the study of the stability of dynamical systems, creating the foundations of the modern theory of the stability in dynamical systems. He considered a continuous dynamical system of the form $\dot{\mathbf{x}}=F(\mathbf{x}), \mathbf{x} \in \mathbb{R}^{n}$, where $F: \mathbb{R}^{n} \rightarrow \mathbb{R}^{n}$ is a $C^{k}$-smooth vector field, for some $k \geq 1$. Near the zero equilibrium $O$, the system of differential equations can be written as $\dot{\mathbf{x}}=A \mathbf{x}+f(\mathbf{x}), O=\{\mathbf{x}=0\}, A=J F(O)$, and $f(O)=J f(O)=0$, where $J$ is the Jacobian matrix of the function. He proved that $O$ is stable if all eigenvalues of the matrix $A$ have negative real parts and $O$ is unstable if there exists an eigenvalue with positive real part. He also studied the case when some eigenvalues have negative real part while the others have zero real part. Moreover, he showed that there exists a two-dimensional invariant surface $M$ passing in the origin when the matrix $A$ has a pair of pure imaginary eigenvalues and the others have negative real parts, in which the equilibrium $O$ of the system is stable whenever $O$ restricted to $M$ is stable. This is precisely what we today know as "centre manifold." Therefore, Lyapunov built the foundations of stability analysis, proving the existence of a centre manifold, formulating the reduction principle and giving stability conditions for equilibrium points.

In discrete dynamical systems of the form $\mathbf{x}_{n+1}=G\left(\mathbf{x}_{n}\right)$, for $n=0,1,2, \ldots$, where $G: \mathbb{R}^{n} \rightarrow \mathbb{R}^{n}$ is a $C^{k}$ smooth mapping, for some $k \geq 1$, which can be written near the origin as $\mathbf{x} \rightarrow A \mathbf{x}+f(\mathbf{x}), O=\{\mathbf{x}=0\}, A=J G(O)$, and $f(O)=J f(O)=0$, the equivalent conclusion to Lyapunov's one is as follows: the fixed point $O$ is locally stable if all the eigenvalues of the matrix $A$ are less than one in absolute value, and $O$ is unstable if there exists at least one of the eigenvalues of $A$ with absolute value greater than one. The centre manifold appears when some of the eigenvalues are one in absolute value, while the others are less than one in absolute value.

We should mention that the reduction principle in the study of the qualitative properties of equilibrium points was completely established in 1964 by Pliss [3]. Three years later, Kelley [4] introduced the modern term of centre manifold and gave a complete proof of the existence of manifolds through equilibrium points in general case.

There exists a parallel between the study of the stability of fixed points in continuous systems and that in discrete systems. A natural extension of the results for continuous 
systems has been deeply investigated for discrete systems. See, for instance, the book from 1979 by Iooss [5], where the author presents a complete list of such results, the book by Carr [6], based on a series of lectures that gives an introduction to the applications of centre manifold theory, and the books by Guckenheimer and Holmes [7] and by Kuznetsov [8], where an exploration of the methods of local bifurcation theory for flows and maps, including centre manifolds and normal forms, is provided.

Some pedagogical examples explaining how to check the stability of fixed points via the centre manifold may be found in several books, as is the example of the book by Elaydi [9], where the author studies a two-dimensional quadratic model and a two-dimensional cubic model, the book by Wiggis [10], where a three-dimensional quadratic mapping is studied, and the book by Zhang [11], where a two-dimensional quadratic map is deeply studied.

In the end of the past century and in the beginnings of the current century, the theory of discrete dynamical systems has developed quicker than ever. The reason may be in the evolution of the computers, as seen in specific software such as Mathematica or Maple, which facilitate the algebraic computations. Nowadays, some nonlinear models can be studied in a much simpler way. Citing some examples, Dektešević et al. [12] studied a two-dimensional quadratic map with two parameters; Guzowska et al. [13] studied a twodimensional rational model with four parameters denominated by logistic competition model; Karydas and Schinas [14] gave a new proof of the center manifold theorem for a system of difference equations; Kulenović and Merino [15] studied a competitive planar map and established conditions that guarantee the existence of an invariant curve when both eigenvalues of the Jacobian of the map are nonzero and at least one of them has absolute value less than one. These results can be applied to many hyperbolic and nonhyperbolic cases. Several applications to planar systems of difference equations are provided in this paper. Luis et al. [16] studied a nonlinear planar model with four parameters denominated planar Ricker competition model and Psarros et al. [17] studied a three-dimensional model with six parameters.

As a consequence of the complexity of the techniques involved in the computations of the centre manifold, the study of the stability of nonhyperbolic fixed points in higher dimensional nonlinear models is usually avoided. The main objective of this survey is precisely to provide the necessary techniques for determining such set in the case of threedimensional models and illustrate them with a concrete nonlinear example, as is the case of the Ricker competition model.

This survey is organized as follows: in Section 2, the principal definitions and results concerning the stability of fixed points in one-dimensional and in two-dimensional discrete models are given. In Section 3, the stability conditions for three-dimensional discrete models using the trace, the determinant, and the sum of the principal minors of the Jacobian matrix are studied. In Section 4, the main tools in order to determine the centre manifold of the system are presented. In Section 5, the stability of the fixed points of the Ricker competition model of three species is studied.
Finally, in Section 6, a conjecture is presented that the local stability conditions of the nontrivial fixed points of the Ricker competition model imply its global stability.

\section{Local Stability in $1 D$ and 2D Systems}

In this section we provide a brief review of the basic results from the literature, related to the study of stability of fixed points in one- and in two-dimensional discrete models.

2.1. One-Dimensional Maps. Consider an interval $I \subseteq \mathbb{R}$ and a map $f: I \rightarrow I$. A point $x^{*} \in \mathbb{R}$ is said to be a fixed point (or equilibrium point) of $f$ if $f\left(x^{*}\right)=x^{*}$, and given $x_{0} \in \mathbb{R}$, we define its orbit $O\left(x_{0}\right)$ as the set of points $O\left(x_{0}\right)=$ $\left\{x_{0}, f\left(x_{0}\right), f^{2}\left(x_{0}\right), f^{3}\left(x_{0}\right), \ldots\right\}$, where $f^{n}=f \circ f^{n-1}$, for $n=$ $1,2, \ldots$

One of the main objectives of the theory of discrete dynamical systems and, in particular, of the stability theory is the study of the behavior of orbits near fixed points, that is, the behavior of solutions of difference equations when the starting points are near equilibrium points. Hence, a basic definition in this field is needed. Let $\mathbb{N}$ denote the set of nonnegative integers.

Definition 1 (local stability). Let $f: I \rightarrow I$ be a map and $x^{*}$ be a fixed point of $f$, where $I$ is an interval of real numbers. Then

(1) the fixed point $x^{*}$ is said to be locally stable if, for any $\epsilon>0$, there exits $\delta>0$ such that, for all $x_{0} \in I$ with $\left|x_{0}-x^{*}\right|<\delta$, we have $\left|f^{n}\left(x_{0}\right)-x^{*}\right|<\epsilon$, for all $n \in \mathbb{N}$. Otherwise, the fixed point $x^{*}$ will be called unstable;

(2) the fixed point $x^{*}$ is said to be attracting if there exists $\eta>0$ such that $\left|x_{0}-x^{*}\right|<\eta$ implies $\lim _{n \rightarrow \infty} f^{n}\left(x_{0}\right)=$ $x^{*}$

(3) the fixed point $x^{*}$ is said to be locally asymptotically stable if it is both stable and attracting. If in the previous item $\eta=\infty$, then $x^{*}$ is said to be globally asymptotically stable.

One of the most effective graphical iteration methods to depict stability of fixed points is the cobweb diagram (also known as stair-step diagram) since it is possible to observe the behavior of the orbit of an initial point near the fixed point. It consists in a sequence of line segments connecting the points in the orbit of an initial point $x_{0}$, that is, a sequence of line segments with end points $\left(x_{0}, x_{1}\right)$ and $\left(x_{1}, x_{1}\right),\left(x_{1}, x_{1}\right)$ and $\left(x_{1}, x_{2}\right),\left(x_{1}, x_{2}\right)$ and $\left(x_{2}, x_{2}\right),\left(x_{2}, x_{2}\right)$ and $\left(x_{2}, x_{3}\right)$, and so forth. On the cobweb plot, a locally stable fixed point corresponds to an inward spiral, while an unstable fixed point is an outward one. It follows from the definition of a fixed point that these spirals will center at a point where the diagonal $y=x$ line crosses the function graph. This technique may be found in many books on discrete dynamical systems or difference equations as are the books by Alligood et al. [18], by Devaney [19], by Elaydi [9, 20], by Zhang [11], and by others, as well. In Figure 1 we present an illustration of the cobweb diagram for the Ricker equation $x_{n+1}=x_{n} e^{r-x_{n}}$, for $n=0,1,2, \ldots$ and $r>0$. 


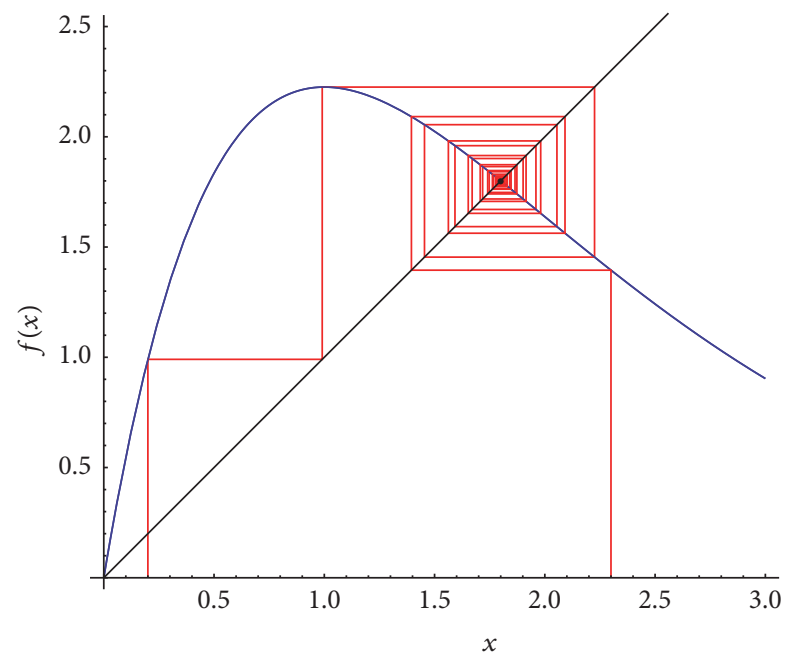

FIGURE 1: Cobweb animation for the Ricker model given by $x_{n+1}=$ $x_{n} e^{r-x_{n}}$. Here we are using $r=1.8$ and one can observe two orbits, the orbit of an initial point $x_{0}=0.2$ and the orbit of an initial point $x_{0}=2.3$. Both orbits converge to the fixed point $x^{*}=1.8$ meaning that the fixed point $x^{*}=1.8$ is locally stable.

However, cobweb diagrams are not the most efficient tool to study local stability. There exists a simple but powerful criterion for knowing the local stability of fixed points. We may divide the fixed points into two categories: hyperbolic and nonhyperbolic. A fixed point $x^{*}$ of a map $f$ is said to be hyperbolic if $\left|f^{\prime}\left(x^{*}\right)\right| \neq 1$. Otherwise, it is nonhyperbolic.

Theorem 2 (see Elaydi [9], page 25). Let $x^{*}$ be a hyperbolic fixed point of a map $f$, where $f$ is continuous and differentiable at $x^{*}$. The following statements hold true:

(1) If $\left|f^{\prime}\left(x^{*}\right)\right|<1$, then $x^{*}$ is locally asymptotically stable.

(2) If $\left|f^{\prime}\left(x^{*}\right)\right|>1$, then $x^{*}$ is unstable.

The stability criteria for nonhyperbolic fixed points are more complex and are summarized in the following theorem (for a complete classification of nonhyperbolic fixed points $\mathrm{cf}$. [9], page 33). Before presenting the criteria we introduce the notion of Schwarzian derivative.

Definition 3 (Schwarzian derivative). The Schwarzian derivative, $S f$, of a function $f$, is defined by

$$
S f(x)=\frac{f^{\prime \prime \prime}(x)}{f^{\prime}(x)}-\frac{3}{2}\left(\frac{f^{\prime \prime}(x)}{f^{\prime}(x)}\right)^{2} .
$$

In particular, when $f^{\prime}\left(x^{*}\right)=-1$, we have $S f\left(x^{*}\right)=-f^{\prime \prime \prime}\left(x^{*}\right)-$ $(3 / 2)\left[f^{\prime \prime}\left(x^{*}\right)\right]^{2}$.

Theorem 4 (see Elaydi [9], pages 28-30). Let $x^{*}$ be a fixed point of a map $f$ and $f^{\prime}, f^{\prime \prime}$, and $f^{\prime \prime \prime}$ be continuous at $x^{*}$.

(1) Let $f^{\prime}\left(x^{*}\right)=1$.

(a) If $f^{\prime \prime}\left(x^{*}\right)>0$, then $x^{*}$ is unstable but semistable from the left. (b) If $f^{\prime \prime}\left(x^{*}\right)<0$, then $x^{*}$ is unstable but semistable from the right.

(c) If $f^{\prime \prime}\left(x^{*}\right)=0$ and $f^{\prime \prime \prime}\left(x^{*}\right)>0$, then $x^{*}$ is unstable.

(d) If $f^{\prime \prime}\left(x^{*}\right)=0$ and $f^{\prime \prime \prime}\left(x^{*}\right)<0$, then $x^{*}$ is locally asymptotically stable.

(2) Let $f^{\prime}\left(x^{*}\right)=-1$.

(a) If $S f\left(x^{*}\right)<0$, then $x^{*}$ is locally asymptotically stable.

(b) If $S f\left(x^{*}\right)>0$, then $x^{*}$ is unstable.

2.2. Two-Dimensional Maps. Definition 1 (local stability) can now be extended to two-dimensional models (or higher dimensional models as well), using an appropriate norm. Since it will follow the same guidelines, we will omit it here.

The local stability of a fixed point $\left(x^{*}, y^{*}\right)$ of the equation

$$
\left(x_{n+1}, y_{n+1}\right)=F\left(x_{n}, y_{n}\right), \quad F=(f, g)
$$

is guaranteed when all the eigenvalues of the Jacobian, $\mathbf{J F}\left(x^{*}, y^{*}\right)$, evaluated at the fixed point $\left(x^{*}, y^{*}\right)$ have magnitude less than one. This is precisely the conclusion of the following result; compare Theorem 4.11 in Elaydi [9], page 221.

Theorem 5 (see Elaydi [9], page 221). Let $F: D \subset \mathbb{R}^{2} \rightarrow$ $\mathbb{R}^{2}$ be a $C^{1}$ map, where $D$ is an open subset of $\mathbb{R}^{2},\left(x^{*}, y^{*}\right)$ a fixed point of $F$, and $\mathbf{J F}=\operatorname{JF}\left(x^{*}, y^{*}\right)$ the Jacobian matrix of $F$ evaluated at the fixed point $\left(x^{*}, y^{*}\right)$ and let $\rho(\mathrm{JF})$ be the spectral radius of the matrix JF. Then the following statements hold true:

(1) If $\rho(\mathbf{J F})<1$, then $\left(x^{*}, y^{*}\right)$ is locally asymptotically stable.

(2) If $\rho(\mathrm{JF})>1$, then $\left(x^{*}, y^{*}\right)$ is unstable.

(3) If $\rho(\mathbf{J F})=1$, then $\left(x^{*}, y^{*}\right)$ may or may not be stable.

The necessary and sufficient conditions can be determined by using the trace, tr, and the determinant, det, of JF as is summarized in the following result; compare Theorem 4.4 in Elaydi [9], page 200:

Theorem 6 (see Elaydi [9], page 200). Let $\left(x^{*}, y^{*}\right)$ be a fixed point of the map $F=(f, g)$ and $\mathbf{J F}=\mathbf{J F}\left(x^{*}, y^{*}\right)$ be the Jacobian matrix evaluated at the fixed point $\left(x^{*}, y^{*}\right)$. Then, $\rho(\mathbf{J F})<1$ if and only if

$$
\left|\operatorname{tr}\left(\mathbf{J F}\left(x^{*}, y^{*}\right)\right)\right|-1<\operatorname{det}\left(\mathbf{J F}\left(x^{*}, y^{*}\right)\right)<1,
$$

where $\rho(\mathbf{J F})$ is the spectral radius of the matrix $\mathbf{J F}$.

Remark 7. Notice that Theorem 6 states that the fixed point $\left(x^{*}, y^{*}\right)$ is locally asymptotically stable if conditions (3) are verified.

The case of nonhyperbolic fixed point is more involved. There are several scenarios according to the eigenvalues 
which are 1 or -1 . When one of the eigenvalues lies in the unit circle and the other eigenvalue is inside the unit circle, it is common to use centre manifold theory $[6,8,20]$ in order to determine the stability of the fixed point, since the dynamics on the centre manifold determines the dynamics of the original system.

In Section 4 we will give the principal definitions and techniques behind the study of nonhyperbolic fixed points for a general dimension. In this specific case of dimension two, a centre manifold is a graph of type $y=h(x)$ (see Theorem 11) on which the dynamics of (2) is given locally by the map on the centre manifold (see Theorem 12).

Since the map on the centre manifold, in this case, is a one-dimensional map, one can then use the results presented in the previous subsection and conclude the stability of the nonhyperbolic fixed point. At this point we refer the pedagogical examples present in Elaydi's book [9, pages 244-248] and in Zhang's book [11, pages 286-288].

\section{Local Stability Conditions in 3D Systems}

An autonomous three-dimensional difference equation is usually given by

$$
\begin{aligned}
& x_{n+1}=f\left(x_{n}, y_{n}, z_{n}\right) \\
& y_{n+1}=g\left(y_{n}, y_{n}, z_{n}\right) \\
& z_{n+1}=h\left(x_{n}, y_{n}, z_{n}\right),
\end{aligned}
$$

where $n=0,1,2, \ldots$ and the functions $f, g$, and $h$ are of class $C^{k}$, with $k$ being as big as we want. For simplicity, let us write system (4) as $\mathbf{x}_{n+1}=F\left(\mathbf{x}_{n}\right)$, where $\mathbf{x}=(x, y, z)$ and $F=(f, g, h)$.

As mentioned before, we will consider an extension of the stability Definition 1 taking a convenient norm in $\mathbb{R}^{3}$. Since one of the main objectives in the field of discrete dynamical systems is the study of the dynamics near the fixed points of $F$, that is, the local stability of the fixed points, a suitable generalization of Theorem 5 states that the fixed point $\mathbf{x}^{*}$ of $F$ is locally asymptotically stable if all the eigenvalues of the Jacobian, $\mathbf{J F}\left(\mathbf{x}^{*}\right)$, evaluated at the fixed point $\mathbf{x}^{*}$ have magnitude less than one. These conditions can be expressed in terms of the determinant of the Jacobian, $\operatorname{det}\left(\operatorname{JF}\left(\mathbf{x}^{*}\right)\right)$, the trace of the Jacobian, $\operatorname{tr}\left(\mathbf{J F}\left(\mathbf{x}^{*}\right)\right)$, and the sum of principal minors of the Jacobian, $\sum_{i=1}^{3} M_{i i}\left(\mathbf{J F}\left(\mathbf{x}^{*}\right)\right)$. Moreover, these three values can be expressed as functions of the eigenvalues of $\mathbf{J F}\left(\mathbf{x}^{*}\right), \lambda_{i}$, for $i=1,2,3$ (cf. Section 2 of Brooks [21]). Namely,

$$
\begin{aligned}
\operatorname{det}\left(\mathbf{J F}\left(\mathbf{x}^{*}\right)\right) & =\lambda_{1} \lambda_{2} \lambda_{3}, \\
\operatorname{tr}\left(\mathbf{J F}\left(\mathbf{x}^{*}\right)\right) & =\lambda_{1}+\lambda_{2}+\lambda_{3}, \\
\sum_{i=1}^{3} M_{i i}\left(\mathbf{J F}\left(\mathbf{x}^{*}\right)\right) & =\lambda_{1} \lambda_{2}+\lambda_{1} \lambda_{3}+\lambda_{2} \lambda_{3} .
\end{aligned}
$$

The characteristic equation of $\mathbf{J F}\left(\mathbf{x}^{*}\right)$ in this case can be given by

$$
\begin{gathered}
\lambda^{3}-\operatorname{tr}\left(\mathbf{J F}\left(\mathbf{x}^{*}\right)\right) \lambda^{2}+\sum_{i=1}^{3} M_{i i}\left(\mathbf{J F}\left(\mathbf{x}^{*}\right)\right) \lambda \\
-\operatorname{det}\left(\mathbf{J F}\left(\mathbf{x}^{*}\right)\right)=0,
\end{gathered}
$$

which has the three eigenvalues as roots. In order to determine conditions that guarantee that the characteristic roots are inside the unit disk, one can use the Schur-Cohn Criterion; compare Theorem 5.1 in Elaydi [20, page 248].

Theorem 8 (see Elaydi, [20] page 248). The zeros of the characteristic polynomial

$$
p(\lambda)=\lambda^{k}+p_{1} \lambda^{k-1}+\cdots+p_{k}
$$

lie inside the unit disk if and only if the following hold:

(i) $p(1)>0$.

(ii) $(-1)^{k} p(-1)>0$.

(iii) The $(k-1) \times(k-1)$ matrices

$$
\begin{aligned}
B_{k-1}^{ \pm}= & \left(\begin{array}{ccccc}
1 & 0 & \cdots & 0 & 0 \\
p_{1} & 1 & \cdots & 0 & 0 \\
\vdots & & \ddots & & \vdots \\
p_{k-3} & p_{k-4} & \cdots & 1 & 0 \\
p_{k-2} & p_{k-3} & \cdots & p_{1} & 1
\end{array}\right) \\
& \left(\begin{array}{ccccc}
0 & 0 & \cdots & 0 & p_{k} \\
0 & 0 & \cdots & p_{k} & p_{k-1} \\
\vdots & & \ddots & & \vdots \\
0 & p_{k} & \cdots & p_{4} & p_{3} \\
p_{k} & p_{k-1} & \cdots & p_{3} & p_{2}
\end{array}\right)
\end{aligned}
$$

are positive innerwise (the determinants of all of its inners are positives), for $k=2,3, \ldots$.

Hence, using the Schur-Cohn Criterion, it follows that the necessary and sufficient conditions for which all the roots of (6) lie inside the unit disk (cf. relation (5.1.17) in Elaydi [9], page 248) are

$$
\begin{aligned}
& \left|\operatorname{tr}\left(\mathbf{J F}\left(\mathbf{x}^{*}\right)\right)+\operatorname{det}\left(\mathbf{J F}\left(\mathbf{x}^{*}\right)\right)\right|<1+\sum_{i=1}^{3} M_{i i}\left(\mathbf{J F}\left(\mathbf{x}^{*}\right)\right) \\
& \left|\sum_{i=1}^{3} M_{i i}\left(\mathbf{J F}\left(\mathbf{x}^{*}\right)\right)-\operatorname{tr}\left(\mathbf{J F}\left(\mathbf{x}^{*}\right)\right) \operatorname{det}\left(\mathbf{J F}\left(\mathbf{x}^{*}\right)\right)\right| \\
& \quad<1-\operatorname{det}^{2}\left(\mathbf{J F}\left(\mathbf{x}^{*}\right)\right) .
\end{aligned}
$$

Thus, conditions (9) imply that the fixed point $\mathbf{x}^{*}$ of (4) is locally asymptotically stable. A remark about conditions (9) is in order. 

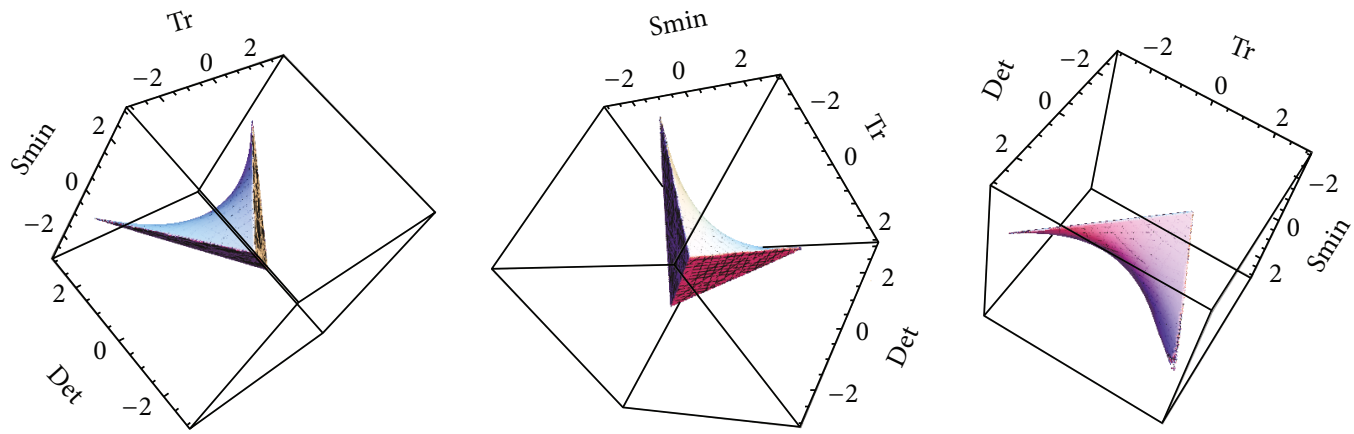

FIGURE 2: Region of local stability of a fixed point in the triple (tr, det, $\sum M_{i i}$ ). Each box represents the same region with different points of view.

Remark 9. Since in most of the concrete cases we deal with linear stability of three-dimensional discrete models depending on parameters, the elements of the Jacobian are most of the times expressed in terms of different parameters. Hence, direct calculations of the eigenvalues through the classical methods are not practical. The main feature of conditions (9) relies in the fact that one can obtain stability conditions, depending on the parameters, without computing the eigenvalues.

Before ending this section, let us look into the region of local stability given by conditions (9). This region, where all the eigenvalues are less than one in magnitude, represents the region of local stability of a fixed point in a three-dimensional discrete dynamical system given by (4). Plotting the region defined by these four conditions in a coordinate system representing the trace $(\mathrm{Tr})$, the determinant (Det), and the sum of the minors (SMin), the result is the picture depicted in Figure 2.

\section{Bifurcation: Stability Using the Centre Manifold Theory}

If some of the eigenvalues of $\mathbf{J F}\left(\mathbf{x}^{*}\right)$ have absolute value equal to one and the other eigenvalues have absolute value less than one, at least one of the conditions of (9) will be replaced by an equality. When this happens we say that a bifurcation occurs. In this case a small smooth change in the parameter values (the bifurcation parameters) of a system causes a sudden qualitative or topological change in its behavior. For a general framework in this area we refer to Kuznetzov's book [8], where the author studies in depth the bifurcation phenomenon in both continuous and discrete dynamical systems.

In certain cases it is possible to study the stability of the nonhyperbolic fixed point via the centre manifold theory since the dynamics on the centre manifold determines the dynamics of the original system.

In the sequel, we will summarize the principal ideas behind the study of stability via the centre manifold theory in discrete dynamical systems. For general framework in the field of difference equations or discrete dynamical systems we refer to the books by Carr [6], Elaydi [9], and
Kuznetzov [8] and for specific results and terminology the papers by Guzowska et al. [13], Luis et al. [16], and Psarros et al. [17].

We will split our analysis into two cases according to the Jacobian matrix at the fixed point which is a block diagonal matrix or an upper (lower) triangular matrix.

4.1. Invariant Manifolds. Let $F: \mathbb{R}^{k} \rightarrow \mathbb{R}^{k}$ be a map such that $F \in C^{2}$ and $F(0)=0$. Then one may write $F$ as a perturbation of a linear map $L$,

$$
F(\mathbf{x})=L \mathbf{x}+R(\mathbf{x}),
$$

where $L$ is a $k \times k$ matrix defined by $L=J F(0), R(0)=0$, and $J R(0)=0$, and $J$ denotes the Jacobian matrix. Now we will introduce special subspaces of $\mathbb{R}^{k}$, called invariant manifolds (cf. Wiggis [10], page 28), that will play a central role in our study of stability and bifurcation.

An invariant manifold is a manifold embedded in its phase space that it is invariant under the dynamical system generated by $F$. A subspace $M$ of $\mathbb{R}^{k}$ is an invariant manifold if, whenever $\mathbf{x} \in M, F^{n}(\mathbf{x}) \in M$, for all $n \in \mathbb{Z}^{+}$. For the linear map $L$, one may split its spectrum $\sigma(L)$ into three sets $\sigma_{s}, \sigma_{u}$, and $\sigma_{c}$, such that $\lambda \in \sigma_{s}$ if $|\lambda|<1, \lambda \in \sigma_{u}$ if $|\lambda|>1$, and $\lambda \in \sigma_{c}$ if $|\lambda|=1$. Associated with these sets, there exist three invariant manifolds (linear subspaces) $E^{\mathcal{s}}, E^{u}$, and $E^{c}$ which are the generalized eigenspaces corresponding to $\sigma_{s}$, $\sigma_{u}$, and $\sigma_{c}$, respectively. It should be noted that some of these subspaces may be trivial subspaces.

The main question here is how to extend this linear theory to nonlinear maps. Corresponding to each of the linear subspaces $E^{s}, E^{u}$, and $E^{c}$ is an invariant manifold: the stable manifold $W^{s}$, the unstable manifold $W^{u}$, and the centre manifold $W^{c}$.

The centre manifold theory is interesting only if $W^{u}=$ $\{0\}$, for, in this case, the dynamics on the centre manifold $W^{c}$ determines the dynamics of the system.

Let $E^{s} \subset \mathbb{R}^{s}, E^{u} \subset \mathbb{R}^{u}$, and $E^{c} \subset \mathbb{R}^{t}$, with $s+u+t=k$. Then one may formally define the above-mentioned invariant manifolds as follows:

$$
\begin{aligned}
W^{s} & =\left\{\mathbf{x} \in \mathbb{R}^{k} \mid F^{n}(\mathbf{x}) \longrightarrow 0 \text { as } n \longrightarrow \infty\right\}, \\
W^{u} & =\left\{\mathbf{x} \in \mathbb{R}^{k} \mid F^{n}(\mathbf{x}) \longrightarrow 0 \text { as } n \longrightarrow-\infty\right\} .
\end{aligned}
$$


Since the stability on the centre manifold $W^{c}$ is not a priori known, we will define it as a manifold of dimension $t$ whose graph is tangent to $E^{c}$ at the origin. It is noteworthy to mention that the centre manifold is not unique, while the stable and unstable manifolds are unique.

The next result summarizes the basic invariant manifolds theory.

Theorem 10 (see Marsden and McCracken [22] page 28). Suppose that $F \in C^{2}$. Then there exist $C^{2}$ stable $W^{s}$ and unstable $W^{u}$ manifolds tangent to $E^{s}$ and $E^{u}$, respectively, at $X=0$ and $C^{1}$ centre manifold $W^{c}$ tangent to $E^{c}$ at $X=0$. Moreover, $W^{c}, W^{s}$, and $W^{u}$ are all invariant manifolds.

4.2. Centre Manifolds: Block Diagonal Matrix. By suitable change of variables, one may represent the map $F$ from the $k$-dimensional equation $\mathbf{x}_{n+1}=F\left(\mathbf{x}_{n}\right)$ as the following system of difference equations:

$$
\begin{aligned}
& x_{n+1}=A x_{n}+f\left(x_{n}, y_{n}\right) \\
& y_{n+1}=B y_{n}+g\left(x_{n}, y_{n}\right) .
\end{aligned}
$$

First we assume that the absolute value of all eigenvalues of the $t \times t$ matrix $A$ is one, and all the eigenvalues of the $s \times s$ matrix $B$ are less than one in magnitude, where $t+s=k$. Moreover,

$$
\begin{gathered}
f(0,0)=0, \\
g(0,0)=0, \\
J f(0,0)=0, \\
J g(0,0)=0 .
\end{gathered}
$$

The following theorem guarantees the existence of a centre manifold, which is a curve $y=h(x)$, on which the dynamics of system (12) is given by the map on the centre manifold. The result may be found in Carr's book [6], in Elaydi's book [9, page 243] (cf. Theorem 5.1), or in Zhang's book [11, page 284] (cf. Theorem 6.7.1).

Theorem 11. There exists a centre manifold for system (12) tangent to $\left\{(x, y) \in \mathbb{R}^{t} \times \mathbb{R}^{s} \mid y=0\right\}$ that can be represented locally as the graph of a function $h: \mathbb{R}^{t} \rightarrow \mathbb{R}^{t}$ such that

$$
\begin{aligned}
W^{c} & =\left\{(x, y) \in \mathbb{R}^{t} \times \mathbb{R}^{s} \mid y=h(x), h(0)=0, \quad J h(0)\right. \\
& =0,|x|<\delta \text { for a sufficiently small } \delta\} .
\end{aligned}
$$

Furthermore, the dynamics restricted to $W^{c}$ is given locally by the equation

$$
x_{n+1}=A x_{n}+f\left(x_{n}, h\left(x_{n}\right)\right), \quad x \in R^{t} .
$$

The main feature of (15) is that its dynamics determine the dynamics of (12). So if $x^{*}=0$ is a stable, asymptotically stable, or unstable fixed point of (15), then the fixed point $\left(x^{*}, y^{*}\right)=(0,0)$ of $(12)$ possesses the corresponding property. This is precisely the conclusion of the following result (cf. Theorem 5.2 in Elaydi [9, page 243] or cf. Theorem 6.7.2 in Zhang [11, page 285]).
Theorem 12. Let $x^{*}=\overrightarrow{0}_{t}$ be a t-dimensional zero fixed point of (15). If $x^{*}=\overrightarrow{0}_{t}$ is a stable, asymptotically stable, or unstable fixed point of (15), then the fixed point $\left(x^{*}, y^{*}\right)$ of (12) is stable, asymptotically stable, or unstable fixed point, respectively, where $y^{*}=\overrightarrow{0}_{s}$ is a s-dimensional zero vector.

To find the map $y=h(x)$, we substitute for $y_{n}$ for $h\left(x_{n}\right)$ in (12) and obtain

$$
\begin{aligned}
& x_{n+1}=A x_{n}+f\left(x_{n}, h\left(x_{n}\right)\right) \\
& y_{n+1}=h\left(x_{n+1}\right)=h\left(A x_{n}+f\left(x_{n}, h\left(x_{n}\right)\right)\right) .
\end{aligned}
$$

But

$y_{n+1}=B y_{n}+g\left(x_{n}, y_{n}\right)=B h\left(x_{n}\right)+g\left(x_{n}, h\left(x_{n}\right)\right)$.

Equating (16) and (17) yields the centre manifold equation

$$
h\left[A x_{n}+f\left(x_{n}, h\left(x_{n}\right)\right)\right]=B h\left(x_{n}\right)+g\left(x_{n}, h\left(x_{n}\right)\right) .
$$

Analogously, if all eigenvalues of $A$ are less than one in magnitude and the absolute value of all the eigenvalues of $B$ is one, one may define the centre manifold $W^{c}$ and obtain the equation

$$
y_{n+1}=B y_{n}+g\left(h\left(y_{n}\right), y_{n}\right),
$$

where $x=h(y)$.

We remark that, in most cases, it is not possible to find an explicit expression for $H$. Since the main interest is to study locally the properties of the system, we may use some approximation. The most common and useful approximation is the power series as it is established in the following result that may be found in Carr's book [6], in Zhang's book [11, page 286], or in a most recent paper of Psarros et al. [17] (cf. Theorem 1.3).

Theorem 13. Let $\psi: \mathbb{R}^{t} \rightarrow \mathbb{R}^{s}$ be a $C^{1}$ map with $\psi\left(\overrightarrow{0}_{t}\right)=\overrightarrow{0}_{s}$ and $J \psi\left(\overrightarrow{0}_{t}\right)=\overrightarrow{0}_{s \times t}$, where $J \psi$ is the Jacobian matrix of $\psi$, and $\overrightarrow{0}_{t}, \overrightarrow{0}_{s}$, and $\overrightarrow{0}_{s \times t}$ are the $1 \times t, 1 \times s$, and $s \times t$ zero matrices, respectively. Suppose that $F(\psi(x))=\left(|x|^{r}\right)$ as $x \rightarrow 0$, for some $r>1$. Then

$$
h(x)=\psi(x)+\left(|x|^{r}\right) .
$$

4.3. Centre Manifolds: Upper (Lower) Triangular Matrix. In working with concrete maps, it is beneficial in certain cases to deal with system where the derivative evaluated at the fixed point is not a block diagonal matrix. Let us now consider, without loss of generality, that the system of difference equations $X_{n+1}=F\left(X_{n}\right)$ is written of the form

$$
\left(\begin{array}{l}
x_{n+1} \\
y_{n+1}
\end{array}\right)=\left(\begin{array}{ll}
A & C \\
0 & B
\end{array}\right)\left(\begin{array}{l}
x_{n} \\
y_{n}
\end{array}\right)+\left(\begin{array}{l}
f\left(x_{n}, y_{n}\right) \\
g\left(x_{n}, y_{n}\right)
\end{array}\right) \text {. }
$$

There are two cases to consider: (i) the absolute value of all the eigenvalues of $A$ is one and all the eigenvalues of $B$ are less than one in absolute value and (ii) the absolute value of all the eigenvalues of $B$ is one and all the eigenvalues of $A$ are 
less than one in absolute value. We will present details only in the first case, since the second follows in a similar way and can be found in Luis et al. [16].

From the general theory in linear algebra, there exists a nonsingular matrix $P$ of the form

$$
P=\left[\begin{array}{cc}
P_{1} & P_{3} \\
0 & P_{2}
\end{array}\right]
$$

such that

$$
\left[\begin{array}{ll}
A & B \\
0 & C
\end{array}\right]=P\left[\begin{array}{ll}
A & 0 \\
0 & B
\end{array}\right] P^{-1}
$$

By suitable changes of variables, $x=P_{1} u+P_{3} v$ and $y=$ $P_{2} v$, one may write

$$
\begin{aligned}
\left(\begin{array}{l}
u_{n+1} \\
v_{n+1}
\end{array}\right)= & \left(\begin{array}{ll}
A & 0 \\
0 & B
\end{array}\right)\left(\begin{array}{l}
u_{n} \\
v_{n}
\end{array}\right) \\
& +P^{-1}\left(\begin{array}{l}
f\left(P_{1} u+P_{3} v, P_{2} v\right) \\
g\left(P_{1} u+P_{3} v, P_{2} v\right)
\end{array}\right) .
\end{aligned}
$$

One can apply the centre manifold theorem and obtain a map $v=h(u)$ with $h(0)=0=h^{\prime}(0)$. The dynamics of (24) is completely determined by the dynamics of the equation

$$
\begin{aligned}
u_{n+1}= & A u_{n}+\widetilde{P}_{1} f\left(P_{1} u_{n}+P_{3} h(u), P_{2} h(u)\right) \\
& +\widetilde{P}_{3} g\left(P_{1} u_{n}+P_{3} h(u), P_{2} h(u)\right),
\end{aligned}
$$

where $\widetilde{P}_{1}, \widetilde{P}_{2}$, and $\widetilde{P}_{3}$ are entries of the matrix

$$
P^{-1}=\left(\begin{array}{cc}
\widetilde{P}_{1} & \widetilde{P}_{3} \\
0 & \widetilde{P}_{2}
\end{array}\right) \text {. }
$$

Hence the curve $v=h(u)$ is the implicit solution of (25) and is the equation of the centre manifold. To find the map $h$ we use the centre manifold equation

$$
\begin{gathered}
h[A u+C h(u)+f(u, h(u))] \\
=B h(u)+g(u, h(u)),
\end{gathered}
$$

and using Taylor approximation, we are able to determine locally the map $h$, as we will illustrate in the next section with the Ricker competition model.

\section{Illustrative Example: Ricker Competition Model}

The three-dimensional Ricker competition model [23, page 31] is usually written as

$$
\begin{aligned}
& x_{1}(n+1)=x_{1}(n) \exp \left(r_{1}-\sum_{i=1}^{3} c_{1 i} x_{i}(n)\right) \\
& x_{2}(n+1)=x_{2}(n) \exp \left(r_{2}-\sum_{i=1}^{3} c_{2 i} x_{i}(n)\right) \\
& x_{3}(n+1)=x_{3}(n) \exp \left(r_{3}-\sum_{i=1}^{3} c_{3 i} x_{i}(n)\right),
\end{aligned}
$$

where $x_{i}(n)$, for $i=1,2,3$, represents the population sizes of species $x_{i}$ at time unit $n$, the parameters $r_{i}$, for $i=1,2,3$, are the inherent exponential growth rates at low densities, and $c_{i, j}$, for $i, j=1,2,3$, are the competition intensity coefficients measuring the effects of intraspecific competition and interspecific competition. More precisely, $c_{i i}$ are the intraspecific competition parameters while $c_{i j}$, for $i \neq j$, are the interspecific competition parameters. Notice that these twelve parameters are assumed to be positive.

For the sake of simplicity, throughout this section we assume that the intraspecific competition parameters are equal to one; that is, $c_{i i}=1$, and the interspecific competition parameters lie on the unit interval; that is, $0<c_{i j}<1$, for $i \neq j$.

System (28) can be represented by the difference equation $\mathbf{x}_{n+1}=F\left(\mathbf{x}_{n}\right)$, where the map $F=\left(f_{1}, f_{2}, f_{3}\right)$ is given by

$$
\begin{aligned}
& F\left(x_{1}, x_{2}, x_{3}\right)=\left(x_{1} e^{r_{1}-x_{1}-c_{12} x_{2}-c_{13} x_{3}}, x_{2} e^{r_{2}-x_{2}-c_{21} x_{1}-c_{23} x_{3}},\right. \\
& \left.x_{3} e^{r_{3}-x_{3}-c_{31} x_{1}-c_{32} x_{2}}\right) .
\end{aligned}
$$

The map $F$ has eight fixed points, namely, the origin $O=$ $(0,0,0)$, three fixed points on the axes $E_{1}=\left(r_{1}, 0,0\right), E_{2}=$ $\left(0, r_{2}, 0\right)$, and $E_{3}=\left(0,0, r_{3}\right)$, three fixes points in each of the planes $E_{12}=\left(\left(r_{1}-c_{12} r_{2}\right) /\left(1-c_{12} c_{21}\right),\left(r_{2}-c_{21} r_{1}\right) /\left(1-c_{12} c_{21}\right), 0\right)$, $E_{13}=\left(\left(r_{1}-c_{13} r_{3}\right) /\left(1-c_{13} c_{31}\right), 0,\left(r_{3}-c_{31} r_{1}\right) /\left(1-c_{13} c_{31}\right)\right)$, and $E_{23}=\left(0,\left(r_{2}-c_{23} r_{3}\right) /\left(1-c_{23} c_{32}\right),\left(r_{3}-c_{32} r_{2}\right) /\left(1-c_{23} c_{32}\right)\right)$, and a possible interior fixed point given by

$$
\begin{aligned}
& E^{*} \\
& =\left(\frac{\left(c_{23} c_{32}-1\right) r_{1}+c_{13}\left(r_{3}-c_{32} r_{2}\right)+c_{12}\left(r_{2}-c_{23} r_{3}\right)}{c_{12}\left(c_{21}-c_{23} c_{31}\right)+c_{23} c_{32}+c_{13}\left(c_{31}-c_{21} c_{32}\right)-1},\right. \\
& \quad \frac{\left(c_{13} c_{31}-1\right) r_{2}+c_{23}\left(r_{3}-c_{31} r_{1}\right)+c_{21}\left(r_{1}-c_{13} r_{3}\right)}{c_{12}\left(c_{21}-c_{23} c_{31}\right)+c_{23} c_{32}+c_{13}\left(c_{31}-c_{21} c_{32}\right)-1}, \\
& \\
& \left.\quad \frac{c_{32} r_{2}+c_{31}\left(r_{1}-c_{12} r_{2}\right)+c_{21}\left(c_{12} r_{3}-c_{32} r_{1}\right)-r_{3}}{c_{12}\left(c_{21}-c_{23} c_{31}\right)+c_{23} c_{32}+c_{13}\left(c_{31}-c_{21} c_{32}\right)-1}\right) .
\end{aligned}
$$

Since we are mainly interested in population dynamics, we always assume that these fixed points are nonnegative.

First, let us have a look in the dynamics in lower dimensions. In the absence of two species, say $x_{2}$ and $x_{3}$, the dynamics of system (28) is the same as the one given by the one-dimensional equation $x_{n+1}=x_{n} e^{r_{1}-x_{n}}=f\left(x_{n}\right)$. This equation has two fixed points, the origin, which is always unstable, and a positive fixed point $x^{*}=r_{1}$, which is globally asymptotically stable when $0<r_{1} \leq 2$. At $r_{1}=2$, a period-doubling bifurcation occurs since $f^{\prime}(2)=-1$. This means that $x^{*}=r_{1}$ becomes an unstable fixed point and a new locally asymptotically stable 2-periodic cycle is born. At $r_{1} \approx 2.512$, a new period-doubling bifurcation occurs. The 2-periodic cycle becomes unstable and a new locally asymptotically stable 4-periodic cycle is born. Moreover, this scenario of period-doubling bifurcation route to chaos will continue as $r_{1}$ increases. 
Now, in the absence of one species, say species $x_{3}$, the dynamics of system (28) is the same as the one of the twodimensional system

$$
\begin{aligned}
& x_{1}(n+1)=x_{1}(n) \exp \left(r_{1}-x_{1}(n)-c_{12} x_{2}(n)\right) \\
& x_{2}(n+1)=x_{2}(n) \exp \left(r_{2}-c_{21} x_{1}(n)-x_{2}(n)\right), \\
& \text { for } n=0,1,2, \ldots .
\end{aligned}
$$

The local dynamics and principal properties of the four fixed points of (31) have been studied in depth by Luis et al. [16] and is shortly as follows:

(1) The origin is always an unstable fixed point.

(2) The point $\left(r_{1}, 0\right)$ is an exclusion fixed point in the $x$ axis which is locally asymptotically stable if and only if $0<r_{1} \leq 2$ and $r_{2}<c_{21} r_{1}$.

(3) The point $\left(0, r_{2}\right)$ is an exclusion fixed point in the $y$ axis which is locally asymptotically stable if and only if $0<r_{2} \leq 2$ and $r_{1}<c_{12} r_{2}$.

(4) The point $\left(x_{1}^{*}, x_{2}^{*}\right)=\left(\left(r_{1}-c_{12} r_{2}\right) /\left(1-c_{12} c_{21}\right),\left(r_{2}-\right.\right.$ $\left.\left.c_{21} r_{1}\right) /\left(1-c_{12} c_{21}\right)\right)$ is a positive fixed point in the interior of the first quadrant, which is locally asymptotically stable, if and only if

$$
\begin{aligned}
& 4\left(c_{12} c_{21}-1\right)+2\left(1-c_{12}\right) r_{2}+2\left(1-c_{21}\right) r_{1} \\
& \leq\left(c_{12} r_{2}-r_{1}\right)\left(c_{21} r_{1}-r_{2}\right) \\
& \quad<\left(1-c_{12}\right) r_{2}+\left(1-c_{21}\right) r_{1} .
\end{aligned}
$$

The stability regions, in the parameter space $O r_{1} r_{2}$, of the nontrivial fixed points, are denoted by $R_{1}, Q_{1}$, and $S_{1}$ and are depicted in Figure 3. The region $R_{1}$ is the stability region of $\left(r_{1}, 0\right)$, the region $Q_{1}$ is the stability region of $\left(0, r_{2}\right)$, and the region $S_{1}$ is the stability region of $\left(x_{1}^{*}, x_{2}^{*}\right)$.

More precisely, the region $S_{1}$ is the region in the $r_{1} O r_{2}$ plane bounded by the lines $r_{2}=r_{1} / c_{12}$ and $r_{2}=c_{21} r_{1}$ and the curve $\gamma_{1}$, where $\gamma_{1}$ is part of a branch of the hyperbola defined by

$$
\begin{aligned}
c_{21} r_{1}^{2} & +2\left(1-c_{21}\right) r_{1}-\left(1+c_{12} c_{21}\right) r_{1} r_{2}+2\left(1-c_{12}\right) r_{2} \\
& +c_{12} r_{2}^{2}+4\left(c_{12} c_{21}-1\right)=0 .
\end{aligned}
$$

Notice that the positive equilibrium point exists if and only if

$$
\begin{aligned}
& c_{12} r_{2}<r_{1}, \\
& c_{21} r_{1}<r_{2}
\end{aligned}
$$

or

$$
\begin{aligned}
& c_{12} r_{2}>r_{1}, \\
& c_{21} r_{1}>r_{2} .
\end{aligned}
$$

It follows that (34) implies that $c_{12} c_{21}<1$ while (35) implies $c_{12} c_{21}>1$. When $c_{12} c_{21}>1$, the coexistence equilibrium point is a saddle and the asymptotic

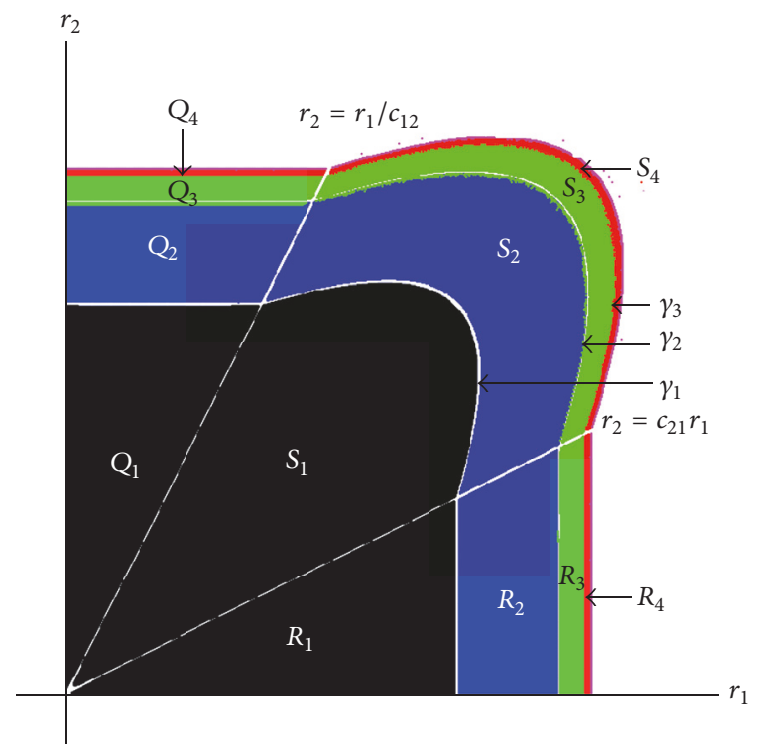

FIgURE 3: The stability regions and the bifurcation scenario of the two-dimensional Ricker competition model (31), in the parameter space $O r_{1} r_{2}$, when the competition parameters $c_{12}$ and $c_{21}$ are fixed such that $c_{12} c_{21}<1$. The region $S_{1}$ is the stability region of the coexistence equilibrium point $\left(x_{1}^{*}, x_{2}^{*}\right)$, the region $R_{1}$ is the stability region of the exclusion fixed point $\left(r_{1}, 0\right)$, and the region $Q_{1}$ is the stability region of the exclusion fixed point $\left(0, r_{2}\right)$. A perioddoubling bifurcation scenario occurs (in the coexistence case) as we cross from region $S_{1}$ to region $S_{2}$, from region $S_{2}$ to region $S_{3}$, and so on. Similarly, one has a period-doubling scenario (in the exclusion case) in the $x$-axis as we cross from region $R_{1}$ to region $R_{2}$, from region $R_{2}$ to region $R_{3}$, and so on. Similar phenomenon occurs in regions $Q_{i}$, for $i=1,2, \ldots$

attractor of an orbit of (31) depends on its initial conditions (see [24] for specific details of this phenomenon in population dynamics). If $c_{12} c_{21}=1$, system (31) has no coexistence equilibrium point. Henceforth, we shall assume that $c_{12} c_{21}<1$ and it means that the interspecific competition is less than the intraspecific competition since $c_{12} c_{21}<c_{11} c_{22}=1$.

Equivalently, in the region defined by (32) and under assumption (34), the coexistence fixed point is locally asymptotically stable if and only if $(r, s) \in \operatorname{int}\left(S_{1}\right) \cup \gamma_{1}$, where $\operatorname{int}\left(S_{1}\right)$ denotes the interior of the region $S_{1}$.

As mentioned before, in order to study the stability of the fixed points of system (28), we have to determine the Jacobian of $F$ which is given by

$$
\begin{aligned}
& J F\left(x_{1}, x_{2}, x_{3}\right) \\
& =\left(\begin{array}{ccc}
-e^{\Delta_{1}}\left(x_{1}-1\right) & -e^{\Delta_{1}} c_{12} x_{1} & -e^{\Delta_{1}} c_{13} x_{1} \\
-e^{\Delta_{2}} c_{21} x_{2} & -e^{\Delta_{2}}\left(x_{2}-1\right) & -e^{\Delta_{2}} c_{23} x_{2} \\
-e^{\Delta_{3}} c_{31} x_{3} & -e^{\Delta_{3}} c_{32} x_{3} & -e^{\Delta_{3}}\left(x_{3}-1\right)
\end{array}\right),
\end{aligned}
$$

where $\Delta_{1}=r_{1}-x_{1}-c_{12} x_{2}-c_{13} x_{3}, \Delta_{2}=r_{2}-c_{21} x_{1}-x_{2}-c_{23} x_{3}$, and $\Delta_{3}=r_{3}-c_{31} x_{1}-c_{32} x_{2}-x_{3}$. 


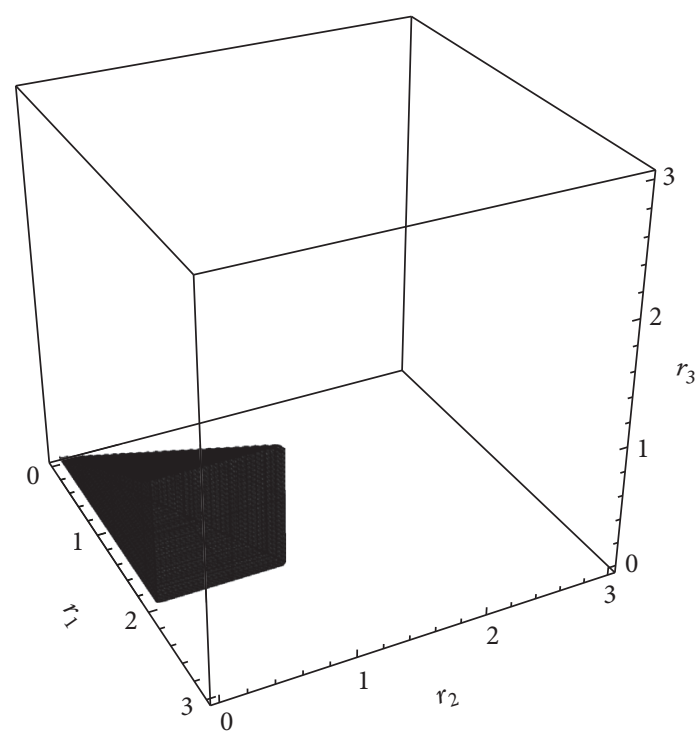

(a)

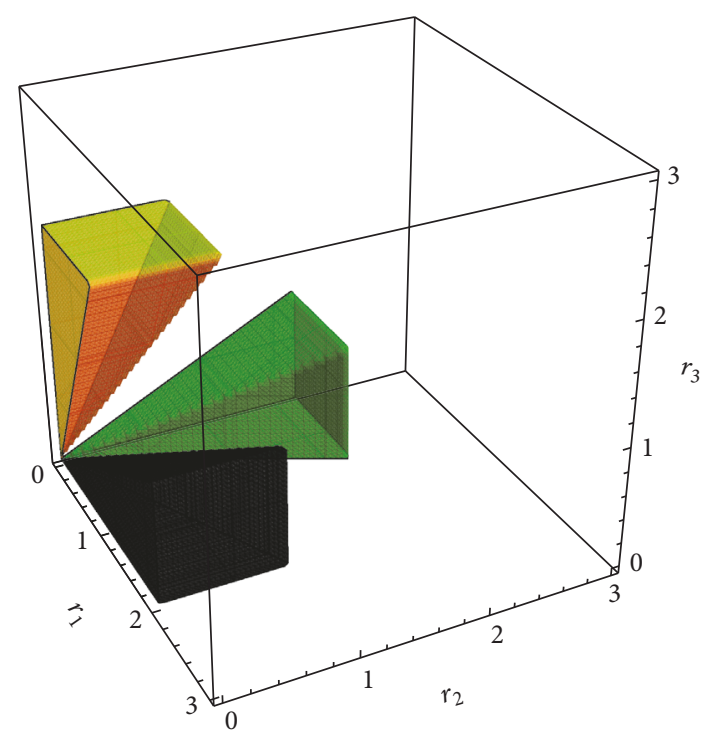

(b)

FIGURE 4: (a) Stability region of the fixed point $E_{1}$, in the parameter space $O r_{1} r_{2} r_{3}$ when $c_{j 1}=0.5$, for $j=2,3$. The projection of this region in the $r_{1} O r_{2}$ plane is the region $R_{1}$ depicted in Figure 3. (b) The stability regions of the fixed points $E_{i}$, for $i=1,2,3$, together in the parameter space $O r_{1} r_{2} r_{3}$ when $c_{i i}=1$ and $c_{i j}=0.5$, for $i \neq j$ and $i, j=1,2,3$.

It is a straightforward computation to see that the origin is an unstable fixed point since the eigenvalues of $J F(O)$ are $e^{r_{i}}>1$, for $i=1,2,3$.

5.1. Fixed Points on the Coordinate Axis. We will reduce our analysis to the fixed point $E_{1}=\left(r_{1}, 0,0\right)$. The stability of the fixed points $E_{i}$, for $i=2,3$, will follow in a similar way.

The Jacobian evaluated at the fixed point $E_{1}$ is given by

$$
J F\left(E_{1}\right)=\left(\begin{array}{ccc}
1-r_{1} & -c_{12} r_{1} & -c_{13} r_{1} \\
0 & e^{r_{2}-c_{21} r_{1}} & 0 \\
0 & 0 & e^{r_{3}-c_{31} r_{1}}
\end{array}\right) .
$$

Clearly, $E_{1}$ is locally asymptotically stable if $0<r_{1}<2$ and $r_{j}<c_{j 1} r_{1}$, for $j=2,3$. This region, in the parameter space of the growth rates (triple $\left(r_{1}, r_{2}, r_{3}\right)$ ), is depicted in Figure 4.

Now, if $r_{1}=2$ and $r_{j}<2 c_{j 1}, j=2,3$ undergoes a perioddoubling bifurcation on the $x$-axis. Under this scenario $\lambda_{1}=$ -1 is an eigenvalue of $J F\left(E_{1}\right)$ and for the other eigenvalues we have $\left|e^{r_{j}-2 c_{j 1}}\right|<1$, for $j=2,3$. Hence, we are in position to study the stability of the fixed point $E_{1}$ via the centre manifold theory.

Shifting the fixed point $E_{1}$ to the origin, it is a straightforward computation to show that system (28) is equivalent to

$$
\begin{aligned}
y_{1}(n+1)= & -y_{1}(n)-2 c_{12} y_{2}(n)-2 c_{13} y_{3}(n) \\
& +\tilde{f}_{1}\left(y_{1}(n), y_{2}(n), y_{3}(n)\right) \\
y_{2}(n+1)= & e^{r_{2}-2 c_{11}} y_{2}(n)+\tilde{f}_{2}\left(y_{1}(n), y_{2}(n), y_{3}(n)\right) \\
y_{3}(n+1)= & e^{r_{3}-2 c_{31}} y_{3}(n) \\
& +\tilde{f}_{3}\left(y_{1}(n), y_{2}(n), y_{3}(n)\right),
\end{aligned}
$$

where

$$
\begin{aligned}
\tilde{f}_{1}\left(y_{1}, y_{2}, y_{3}\right)= & \left(y_{1}+2\right) e^{-c_{12} y_{2}-c_{13} y_{3}-y_{1}}+2 c_{12} y_{2} \\
& +2 c_{13} y_{3}+y_{1}-2, \\
\tilde{f}_{2}\left(y_{1}, y_{2}, y_{3}\right)= & e^{r_{2}} y_{2}\left(e^{-c_{21}\left(y_{1}+2\right)-c_{23} y_{3}-y_{2}}-e^{-2 c_{21}}\right), \\
\tilde{f}_{3}\left(y_{1}, y_{2}, y_{3}\right)= & e^{r_{3}} y_{3}\left(e^{-c_{31}\left(y_{1}+2\right)-c_{32} y_{2}-y_{3}}-e^{-2 c_{31}}\right) .
\end{aligned}
$$

The centre manifold in this case can be represented locally as the graph of a function $\Phi: \mathbb{R}^{2} \rightarrow \mathbb{R}^{2}$ such that

$$
\begin{aligned}
W^{c} & =\left\{\left(y_{1}, y_{2}, y_{3}\right) \in \mathbb{R} \times \mathbb{R}^{2} \mid\left(y_{2}, y_{3}\right)\right. \\
& =\left(\Phi_{1}\left(y_{1}\right), \Phi_{2}\left(y_{1}\right)\right), \Phi(0)=0, J \Phi(0)=0,|y| \\
& <\delta \text { for a sufficiently small } \delta\} .
\end{aligned}
$$

The centre manifold equation (27) is therefore given by

$$
\begin{aligned}
\Phi_{1} & {\left[-y_{1}-2 c_{12} \Phi_{1}\left(y_{1}\right)-2 c_{13} \Phi_{2}\left(y_{1}\right)\right.} \\
& \left.+\tilde{f}_{1}\left(y_{1}, \Phi_{1}\left(y_{1}\right), \Phi_{2}\left(y_{1}\right)\right)\right]=e^{r_{2}-2 c_{1}} \Phi_{1}\left(y_{1}\right) \\
& +\tilde{f}_{2}\left(y_{1}, \Phi_{1}\left(y_{1}\right), \Phi_{2}\left(y_{1}\right)\right) \\
\Phi_{2} & {\left[-y_{1}-2 c_{12} \Phi_{1}\left(y_{1}\right)-2 c_{13} \Phi_{2}\left(y_{1}\right)\right.} \\
& \left.+\tilde{f}_{1}\left(y_{1}, \Phi_{1}\left(y_{1}\right), \Phi_{2}\left(y_{1}\right)\right)\right]=e^{r_{3}-2 c_{31}} \Phi_{2}\left(y_{1}\right) \\
& +\tilde{f}_{3}\left(y_{1}, \Phi_{1}\left(y_{1}\right), \Phi_{2}\left(y_{1}\right)\right),
\end{aligned}
$$

where $\Phi_{i}, i=1,2$, can be represented locally by $\Phi_{i}(u)=$ $\alpha_{i} u^{2}+\beta_{i} u^{3}+O[u]^{4}$. 
Using Mathematica or Maple, we are able to simplify system (41), and using power series one can conclude that (locally) system (41) is equivalent to

$$
\begin{aligned}
& \left(1-e^{r_{2}-2 c_{21}}\right) \alpha_{1} y_{1}^{2}+\left(c_{21} e^{r_{2}-2 c_{21}} \alpha_{1}+4 c_{13} \alpha_{1} \alpha_{2}\right. \\
& \left.+4 c_{12} \alpha_{1}^{2}-\left(1+e^{r_{2}-2 c_{21}}\right) \beta_{1}\right) y_{1}^{3}+O\left[y_{1}\right]^{4}=0 \\
& \left(\alpha_{1}-\alpha_{2} e^{r_{3}-2 c_{31}}\right) y_{1}^{2}+\left(-\beta_{1}+4 c_{12} \alpha_{1}^{2}+4 c_{13} \alpha_{1} \alpha_{2}\right. \\
& \left.+c_{31} e^{r_{3}-2 c_{31}} \alpha_{2}-e^{r_{3}-2 c_{31}} \beta_{2}\right) y_{1}^{3}+O\left[y_{1}\right]^{4}=0
\end{aligned}
$$

In order to have equality, from this system we have to conclude that

$$
\begin{aligned}
& \left(1-e^{r_{2}-2 c_{21}}\right) \alpha_{1}=0 \\
& c_{21} e^{r_{2}-2 c_{21}} \alpha_{1}+4 c_{13} \alpha_{1} \alpha_{2}+4 c_{12} \alpha_{1}^{2}-\left(1+e^{r_{2}-2 c_{21}}\right) \beta_{1} \\
& \quad=0 \\
& \alpha_{1}-\alpha_{2} e^{r_{3}-2 c_{31}}=0 \\
& -\beta_{1}+4 c_{12} \alpha_{1}^{2}+4 c_{13} \alpha_{1} \alpha_{2}+c_{31} e^{r_{3}-2 c_{31}} \alpha_{2}-e^{r_{3}-2 c_{31}} \beta_{2} \\
& \quad=0
\end{aligned}
$$

yielding the trivial solution $\alpha_{1}=\alpha_{2}=\beta_{1}=\beta_{2}=0$.

A straightforward computation shows that locally, the map in (25), in this case, can be given by

$$
f\left(y_{1}\right)=e^{-y_{1}}\left(y_{1}+2\right)-2 .
$$

Since $f^{\prime}(0)=-1$, we have to compute the Schwarzian derivative at the fixed point 0 (cf. Theorem 4 ); that is,

$$
\frac{f^{\prime \prime \prime}(0)}{f^{\prime}(0)}-\frac{3}{2}\left(\frac{f^{\prime \prime}(0)}{f^{\prime}(0)}\right)^{2}=-1<0 .
$$

Hence, the fixed point 0 is locally asymptotically stable.
Consequently, the fixed point $E_{1}$ of system (28) is locally asymptotically stable if $r_{1}=2$ and $r_{j}<2 c_{j 1}$, for $j=2,3$. These specific values of the parameters undergo a perioddoubling bifurcation. As $r_{1}$ increases, the exclusion fixed point $E_{1}$ becomes unstable and an asymptotically stable 2periodic cycle of the form

$$
\left\{\left(\bar{r}_{10}, 0,0\right),\left(\bar{r}_{11}, 0,0\right)\right\}, \quad \bar{r}_{10}<r_{1}<\bar{r}_{11}
$$

is born in the $x$-axis.

From now on, computations are only possible via numerical simulations. One can observe that at $r_{1} \approx 2.512$ a new period-doubling bifurcation occurs. Hence, the exclusion 2periodic cycle on the $x$-axis becomes unstable and a new 4 -periodic cycle in the $x$-axis is born. Further simulations suggest that this scenario of period-doubling bifurcation, in the $x$-axis, will continue route to chaos.

We remark that this period-doubling scenario, in threedimensional mappings, is just a direct observation from the simulations since there is no extension of Sharkovsky's theorem $[25,26]$ for dimension $k \geq 2$.

It remains to study the stability of the fixed point $E_{1}$ when $r_{1}=2, r_{2}=2 c_{21}$, and $r_{3}<2 c_{31}$. Under this restriction on the parameters we have $\lambda_{1}=-1, \lambda_{2}=1$, and $\left|\lambda_{3}\right|<$ 1. The reduced equation on the centre manifold will be a two-dimensional system. Unfortunately, this scenario in twodimensional discrete systems $\left(\widetilde{\lambda}_{1}=-1, \widetilde{\lambda}_{2}=1\right)$ remains as an open problem, and thus we are not able to discuss the stability of the fixed point here.

5.2. Fixed Points on the Planes. Let us now consider the fixed point $E_{12}$ on the plane $x_{1} O x_{2}$. The stability analysis of $E_{13}$ and $E_{23}$ follows in a similar way.

The Jacobian evaluated at the fixed point is given by

$$
J F\left(E_{12}\right)=\left(\begin{array}{ccc}
\frac{1-c_{12} c_{21}+c_{12} r_{2}-r_{1}}{1-c_{12} c_{21}} & \frac{c_{12}\left(c_{12} r_{2}-r_{1}\right)}{1-c_{12} c_{21}} & \frac{c_{13}\left(c_{12} r_{2}-r_{1}\right)}{1-c_{12} c_{21}} \\
\frac{c_{21}\left(c_{21} r_{1}-r_{2}\right)}{1-c_{12} c_{21}} & \frac{1-c_{12} c_{21}+r_{1} c_{21}-r_{2}}{1-c_{12} c_{21}} & \frac{c_{23}\left(c_{21} r_{1}-r_{2}\right)}{1-c_{12} c_{21}} \\
0 & 0 & e^{\left(\left(c_{21} c_{32}-c_{31}\right) r_{1}+\left(c_{31} c_{12}-c_{32}\right) r_{2}+\left(1-c_{21} c_{12}\right) r_{3}\right) /\left(1-c_{12} c_{21}\right)}
\end{array}\right)
$$

It is a straightforward computation to see that the conditions of local stability are given by

$$
\begin{aligned}
& 4\left(c_{12} c_{21}-1\right)+2\left(1-c_{12}\right) r_{2}+2\left(1-c_{21}\right) r_{1} \\
& \quad<\left(c_{12} r_{2}-r_{1}\right)\left(c_{21} r_{1}-r_{2}\right) \\
& \quad<\left(1-c_{12}\right) r_{2}+\left(1-c_{21}\right) r_{1}, \\
& \left(c_{21} c_{32}-c_{31}\right) r_{1}+\left(c_{31} c_{12}-c_{32}\right) r_{2}+\left(1-c_{21} c_{12}\right) r_{3} \\
& \quad<0 .
\end{aligned}
$$

In Figure 5 a prototype of this region is depicted in the triple $\left(r_{1}, r_{2}, r_{3}\right)$ when $c_{j i}=0.5$, for $i \neq j$.

Considering the equality

$$
\begin{aligned}
& 4\left(c_{12} c_{21}-1\right)+2\left(1-c_{12}\right) r_{2}+2\left(1-c_{21}\right) r_{1} \\
& =\left(c_{12} r_{2}-r_{1}\right)\left(c_{21} r_{1}-r_{2}\right)
\end{aligned}
$$

and keeping the other relations, the eigenvalues of $J F\left(E_{12}\right)$ are such that $\lambda_{1}=-1$ and $\left|\lambda_{i}\right|<1$, for $i=2,3$. Hence, we are in position to use the centre manifold theorem. Following the techniques employed in the previous subsection, one can conclude that $E_{12}$ is a locally asymptotically stable 


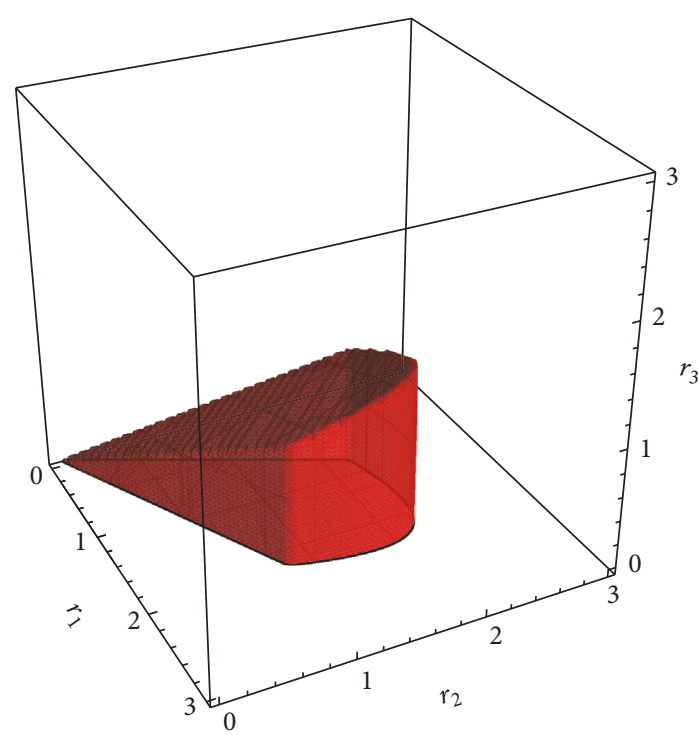

(a)

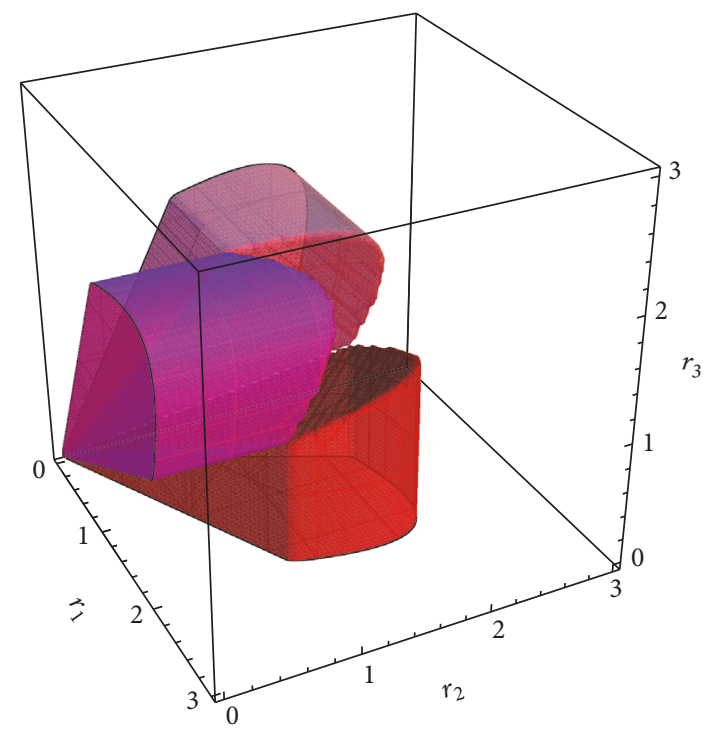

(b)

Figure 5: (a) Stability region of the exclusion fixed point $E_{12}$ in the parameter space $O r_{1} r_{2} r_{3}$ when $c_{j i}=0.5$ for $i \neq j$. The projection of this region in the plane $r_{1} O r_{2}$ is the region $S_{1}$ depicted in Figure 3. (b) Stability regions of $E_{12}, E_{13}$, and $E_{23}$, together in the parameter space $O r_{1} r_{2} r_{3}$ when $c_{i i}=1$ and $c_{i j}=0.5$, for $i \neq j$ and $i, j=1,2,3$.

fixed point on the plane $x_{1} O x_{2}$ under these restrictions on the parameters. Notice that, in the parameter space, the projection of this region in the $r_{1} O r_{2}$ plane is part of a branch of an hyperbola as described in the beginning of this section and can be observed in Figure 3 by $\gamma_{1}$.

This means that this specific region undergoes a perioddoubling bifurcation on the $x_{1} O x_{2}$ plane. Hence, as the parameters increase, the fixed point $E_{12}$ becomes unstable and a locally asymptotically stable 2 -periodic cycle of the form

$$
\left\{\left(\bar{x}_{0}, \bar{y}_{0}, 0\right),\left(\bar{x}_{1}, \bar{y}_{1}, 0\right)\right\}
$$

is born on the $x_{1} O x_{2}$ plane. As in the precedent case, from now on, computations are only possible via numerical simulations. These simulations suggest a scenario of perioddoubling bifurcation on the $x_{1} O x_{2}$ plane route to chaos.

We should mention that if we replace the inequality signs by equality on (48) and condition (49) is satisfied, then $\lambda_{1}=-1, \lambda_{2}=1$, and $\left|\lambda_{3}\right|<1$ are the eigenvalues of
$J F\left(E_{12}\right)$. Unfortunately, we are not able to use here the centre manifold theorem, as mentioned at the end of the previous subsection.

5.3. Positive Fixed Point. We now consider the positive fixed point $E^{*}$ known in population dynamics as the coexistence fixed point. Due to the great number of parameters involved and for the sake of simplicity, we will study the symmetric case, that is, when $c_{i j}=c$, for all $i \neq j$. The fixed point $E^{*}=$ $\left(x_{1}^{*}, x_{2}^{*}, x_{3}^{*}\right)$ becomes in this case

$$
\begin{aligned}
E^{*}= & \left(\frac{c\left(r_{2}+r_{3}\right)-(c+1) r_{1}}{(c-1)(2 c+1)}, \frac{c r_{1}-(c+1) r_{2}+c r_{3}}{(c-1)(2 c+1)},\right. \\
& \left.\frac{c r_{1}+c r_{2}-(c+1) r_{3}}{(c-1)(2 c+1)}\right)
\end{aligned}
$$

and the derivative at $E^{*}$ is given by

$$
\begin{aligned}
& J F\left(E^{*}\right) \\
& =\left(\begin{array}{ccc}
\frac{-2 c^{2}+r_{2} c+r_{3} c+c-(c+1) r_{1}+1}{-2 c^{2}+c+1} & \frac{c\left((c+1) r_{1}-c\left(r_{2}+r_{3}\right)\right)}{(c-1)(2 c+1)} & \frac{c\left((c+1) r_{1}-c\left(r_{2}+r_{3}\right)\right)}{(c-1)(2 c+1)} \\
\frac{c\left(-c r_{1}+(c+1) r_{2}-c r_{3}\right)}{(c-1)(2 c+1)} & \frac{-2 c^{2}+r_{1} c+r_{3} c+c-(c+1) r_{2}+1}{-2 c^{2}+c+1} & \frac{c\left(-c r_{1}+(c+1) r_{2}-c r_{3}\right)}{(c-1)(2 c+1)} \\
\frac{c\left(-c r_{1}-c r_{2}+(c+1) r_{3}\right)}{(c-1)(2 c+1)} & \frac{c\left(-c r_{1}-c r_{2}+(c+1) r_{3}\right)}{(c-1)(2 c+1)} & \frac{-2 c^{2}+r_{1} c+r_{2} c-r_{3} c+c-r_{3}+1}{-2 c^{2}+c+1}
\end{array}\right) .
\end{aligned}
$$


For this matrix we have

$$
\begin{aligned}
& \operatorname{tr}\left(J F\left(E^{*}\right)\right)=\frac{-6 c+r_{1}+r_{2}+r_{3}-3}{2 c+1}, \\
& \sum_{i=1}^{3} M_{i i}\left(J F\left(E^{*}\right)\right) \\
& \quad=\frac{3 P_{0}+2 P_{1}\left(r_{1}+r_{2}+r_{3}\right)+P_{2}\left(r_{1}^{2}+r_{2}^{2}+r_{3}^{2}\right)}{P_{0}} \\
& \quad+\frac{P_{4}\left(r_{1} r_{2}+r_{1} r_{3}+r_{2} r_{3}\right)}{P_{0}}, \\
& \operatorname{det}\left(J F\left(E^{*}\right)\right) \\
& \quad=-\frac{P_{0}+\sum_{i=1}^{3} P_{i}\left(r_{1}^{i}+r_{2}^{i}+r_{3}^{i}\right)+P_{4}\left(r_{1} r_{2}+r_{1} r_{3}+r_{2} r_{3}\right)}{P_{0}} \\
& \quad-\frac{P_{5}\left(r_{1}\left(r_{2}^{2}+r_{3}^{2}\right)+r_{2}\left(r_{1}^{2}+r_{3}^{2}\right)+r_{3}\left(r_{1}^{2}+r_{2}^{2}\right)\right)+P_{6} r_{1} r_{2} r_{3}}{P_{0}},
\end{aligned}
$$

where

$$
\begin{aligned}
& P_{0}=4 c^{3}-3 c-1, \\
& P_{1}=-2 c^{2}+c+1, \\
& P_{2}=c^{3}+3 c^{2}+2 c \\
& P_{3}=c^{3}+c^{2}, \\
& P_{4}=-\left(2 c^{3}+2 c^{2}+c+1\right), \\
& P_{5}=-\left(c^{3}+c^{2}+c\right) \\
& P_{6}=1+3 c+6 c^{2}+2 c^{3} .
\end{aligned}
$$

we shift the fixed point to the origin. System (28) is now equivalent to

$$
Y(n+1)=J Y(n)+G(Y(n)),
$$

where

$$
\begin{aligned}
& Y(n)=\left(\begin{array}{l}
y_{1}(n) \\
y_{2}(n) \\
y_{3}(n)
\end{array}\right), \\
& J=\left(\begin{array}{lll}
J_{11} & J_{12} & J_{13} \\
J_{21} & J_{22} & J_{23} \\
J_{31} & J_{32} & J_{33}
\end{array}\right), \\
& G(Y(n))=\left(\begin{array}{l}
g_{1}(Y(n)) \\
g_{2}(Y(n)) \\
g_{3}(Y(n))
\end{array}\right),
\end{aligned}
$$

$$
\begin{aligned}
& y_{1}=x_{1}-x_{1}^{*}, \\
& y_{2}=x_{2}-x_{2}^{*}, \\
& y_{3}=x_{3}-x_{3}^{*},
\end{aligned}
$$

$$
=\left(\begin{array}{ccc}
\frac{-2 c^{2}+c\left(r_{2}+r_{3}\right)+(c+1)\left(1-r_{1}\right)}{-2 c^{2}+c+1} & \frac{c\left((c+1) r_{1}-c\left(r_{2}+r_{3}\right)\right)}{(c-1)(2 c+1)} & \frac{c\left((c+1) r_{1}-c\left(r_{2}+r_{3}\right)\right)}{(c-1)(2 c+1)} \\
-\frac{c\left(c r_{1}-(c+1) r_{2}+c r_{3}\right)}{(c-1)(2 c+1)} & \frac{-2 c^{2}+r_{1} c+r_{3} c+c-(c+1) r_{2}+1}{-2 c^{2}+c+1} & -\frac{c\left(c r_{1}-(c+1) r_{2}+c r_{3}\right)}{(c-1)(2 c+1)} \\
-\frac{c\left(c r_{1}+c r_{2}-(c+1) r_{3}\right)}{(c-1)(2 c+1)} & -\frac{c\left(c r_{1}+c r_{2}-(c+1) r_{3}\right)}{(c-1)(2 c+1)} & \frac{-2 c^{2}+r_{1} c+r_{2} c-r_{3} c+c-r_{3}+1}{-2 c^{2}+c+1}
\end{array}\right),
$$

$g_{i}\left(y_{1}, y_{2}, y_{3}\right)=f_{i}\left(y_{1}+x_{1}^{*}, y_{2}+x_{2}^{*}, y_{3}+x_{3}^{*}\right)-x_{i}^{*}-\sum_{j=1}^{3} J_{i j} y_{j}, \quad i=1,2,3$. 


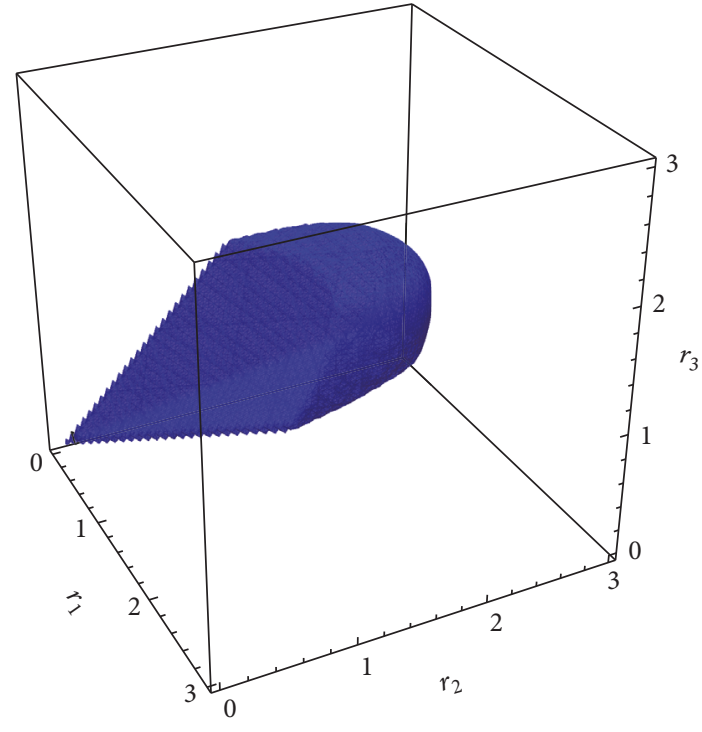

FIGURE 6: Stability region of the positive fixed point $E^{*}$ in the parameter space $O r_{1} r_{2} r_{3}$ when $c_{i i}=1$ and $c_{j i}=0.5$, for $i \neq j$ and $i, j=1,2,3$.

Since $J$ is nonsingular, there exist a diagonal matrix $\Lambda$ and the matrices $P$ and $P^{-1}$ such that $J=P \Lambda P^{-1}$. Making a new change of variables $Y=P Z$, where $Z=\left(z_{1}, z_{2}, z_{3}\right)$, one can write (58) as

$$
Z(n+1)=\Lambda Z(n)+H(Z(n))
$$

where

$$
\begin{aligned}
Z(n) & =\left(\begin{array}{l}
z_{1}(n) \\
z_{2}(n) \\
z_{3}(n)
\end{array}\right), \\
\Lambda & =\left(\begin{array}{ccc}
-1 & 0 & 0 \\
0 & \lambda_{2} & 0 \\
0 & 0 & \lambda_{3}
\end{array}\right), \\
H(Z(n)) & =P^{-1} G(P \times Z(n)) .
\end{aligned}
$$

The centre manifold in this case can be represented locally as the graph of a function $\Phi: \mathbb{R}^{2} \rightarrow \mathbb{R}^{2}$ such that

$$
\begin{aligned}
W^{c} & =\left\{\left(z_{1}, z_{2}, z_{3}\right) \in \mathbb{R} \times \mathbb{R}^{2} \mid\left(z_{2}, z_{3}\right)\right. \\
& =\left(\Phi_{1}\left(z_{1}\right), \Phi_{2}\left(z_{1}\right)\right), \Phi(0)=0, D \Phi(0) \\
& \left.=0,\left|z_{1}\right|<\delta \text { for a sufficiently small } \delta\right\} .
\end{aligned}
$$

Therefore, the centre manifold equation (27) is given by

$$
\begin{aligned}
\Phi_{1} & {\left[-z_{1}+h_{1}\left(z_{1}, \Phi_{1}\left(z_{1}\right), \Phi_{2}\left(z_{1}\right)\right)\right] } \\
& =\lambda_{2} \Phi_{1}\left(z_{1}\right)+h_{2}\left(z_{1}, \Phi_{1}\left(z_{1}\right), \Phi_{2}\left(z_{1}\right)\right) \\
\Phi_{2} & {\left[-z_{1}+h_{1}\left(z_{1}, \Phi_{1}\left(z_{1}\right), \Phi_{2}\left(z_{1}\right)\right)\right] } \\
& =\lambda_{3} \Phi_{2}\left(z_{1}\right)+h_{3}\left(z_{1}, \Phi_{1}\left(z_{1}\right), \Phi_{2}\left(z_{1}\right)\right),
\end{aligned}
$$

where $\Phi_{i}, i=1,2$, can be represented locally by $\Phi_{i}(u)=$ $\alpha_{i} u^{2}+\beta_{i} u^{3}+O[u]^{4}$ and

$$
h_{i}\left(z_{1}, z_{2}, z_{3}\right)=\sum_{j=1}^{3} \widetilde{P}_{i j} g_{j}\left(z_{1}, z_{2}, z_{3}\right), \quad i=1,2,3 \text {, }
$$

where $\widetilde{P}_{i j}$ are the entries of the matrix $P^{-1}$.

Unfortunately, at this stage the computations are long and we are not able to find explicitly the matrices $P, \Lambda$, and $P^{-1}$ such that $J=P \Lambda P^{-1}$. However, we are able to find it numerically. Hence, we will illustrate the study with a concrete example. Let $c=0.5$ and $r_{2}=r_{3}=1.5$ (the choice of these values is made considering the region obtained in Figure 6). Substituting in

$$
\operatorname{tr}\left(J F\left(E^{*}\right)\right)+\operatorname{det}\left(J F\left(E^{*}\right)\right)-\sum_{i=1}^{3} M_{i i}\left(J F\left(E^{*}\right)\right)=1,
$$

we obtain $r_{1} \approx 2.1547$. Under these values of the parameters, the matrices are given by

$$
\begin{aligned}
P & =\left(\begin{array}{ccc}
-0.9769 & 0 & -0.6676 \\
-0.1511 & -0.7071 & 0.5265 \\
-0.1511 & 0.7071 & 0.5265
\end{array}\right), \\
\Lambda & =\left(\begin{array}{ccc}
-1 . & 0 & 0 \\
0 & 0.7887 & 0 \\
0 & 0 & 0.634
\end{array}\right),
\end{aligned}
$$

$$
P^{-1}=\left(\begin{array}{ccc}
-0.8558 & -0.5425 & -0.5425 \\
0 & -0.7071 & 0.7071 \\
-0.2457 & 0.794 & 0.794
\end{array}\right) \text {. }
$$

And the functions $h_{i}\left(z_{1}, z_{2}, z_{3}\right)$, for $i=1,2,3$, are

$$
\begin{aligned}
h_{1} & \left(z_{1}, z_{2}, z_{3}\right)=1.9409+z_{1} \\
& +\left(0.836 z_{1}+0.5713 z_{3}-1.4823\right) e^{1.128 z_{1}+0.1411 z_{3}} \\
& +\left(0.082 z_{1}-0.3836 z_{2}-0.2856 z_{3}-0.2293\right) \\
& \cdot e^{0.7151 z_{1}-0.3536 z_{2}-0.4559 z_{3}} \\
& +\left(0.082 z_{1}+0.3836 z_{2}-0.2856 z_{3}-0.2293\right) \\
& \cdot e^{0.7151 z_{1}+0.3536 z_{2}-0.4559 z_{3}}, \\
h_{2} & \left(z_{1}, z_{2}, z_{3}\right)=-0.7887 z_{2} \\
& +\left(0.1069 z_{1}+0.5 z_{2}-0.3723 z_{3}-0.2989\right) \\
& \cdot e^{0.7151 z_{1}+0.3536 z_{2}-0.456 z_{3}} \\
& +\left(-0.1069 z_{1}+0.5 z_{2}+0.3723 z_{3}+0.2989\right) \\
& \cdot e^{0.7151 z_{1}-0.3536 z_{2}-0.4559 z_{3}},
\end{aligned}
$$




$$
\begin{aligned}
h_{3} & \left(z_{1}, z_{2}, z_{3}\right)=-0.634 z_{3}-0.2456 \\
& +\left(0.24 z_{1}+0.164 z_{3}-0.4255\right) e^{1.128 z_{1}+0.1411 z_{3}} \\
& +\left(0.3356-0.12 z_{1}+0.5614 z_{2}+0.418 z_{3}\right) \\
& \cdot e^{0.7151 z_{1}-0.3536 z_{2}-0.456 z_{3}} \\
& +\left(-0.12 z_{1}-0.5614 z_{2}+0.418 z_{3}+0.336\right) \\
& \cdot e^{0.7151 z_{1}+0.354 z_{2}-0.456 z_{3}}
\end{aligned}
$$

Using Taylor series and solving system (63), one can conclude that the dynamics of the system is given locally by the dynamics of $z_{1}(n+1)=\widetilde{h}\left(z_{1}(n)\right)$, where

$$
\begin{aligned}
\tilde{h}\left(z_{1}\right)= & e^{0.7151 z_{1}}\left(0.164 z_{1}-0.4586\right) \\
& +e^{1.128 z_{1}}\left(0.836 z_{1}-1.4823\right)+1.9409 .
\end{aligned}
$$

Notice that this corresponds to the equation on the centre manifold that is generically given by (15).

Since $\tilde{h}^{\prime}(0)=-1$, we have to use Theorem 4 . The Schwarzian derivative of $\tilde{h}$ at 0 is $\approx-1.1476<0$. It follows from Theorem 4 that, in this particular case, the fixed point is locally asymptotically stable.

We remark that, due to the difficulties encountered in finding the matrix $P$, one can write a script in Mathematica or Maple to automatically obtain, via numerical approximation, the map $\widetilde{h}$ in each case.

\section{Conjecture}

One of the main questions in population dynamics is under what conditions does local stability of a fixed point imply its global asymptotic stability?

One of the most influential models in population dynamics is the one-dimensional Ricker equation given by $x_{n+1}=$ $x_{n} e^{r-x_{n}}, r>0$. The local stability condition of the positive fixed point $x^{*}=r, 0<r \leq 2$, implies global stability of $x^{*}$; that is, all solutions of the difference equation $x_{n+1}=x_{n} e^{r-x_{n}}$, starting at an initial condition $x_{0}>0$, converge to $x^{*}=r$ whenever $0<r \leq 2$. In order to establish this conclusion one can use Coppell's Theorem since $x^{*}=r$ is the unique positive fixed point of the map.

Theorem 14 (see Coppell [27]). Let $I=[a, b] \subseteq \mathbb{R}$ and $f$ : $I \rightarrow I$ be a continuous map. If the equation $f(f(x))=x$ has no roots, with the possible exception of the roots of the equation $f(x)=x$, then every orbit under the map $f$ converges to a fixed point.

In planar models this question of global stability is more involved since we do not have an analogue of Coppell's Theorem, but alternative methods do exist. For instance, Balreira et al. [28], used the Singularity Theory of
Whitney [29] and proved that the local stability conditions (32) of the positive fixed point

$$
\left(x_{1}^{*}, x_{2}^{*}\right)=\left(\frac{r_{1}-c_{12} r_{2}}{1-c_{12} c_{21}}, \frac{r_{2}-c_{21} r_{1}}{1-c_{12} c_{21}}\right)
$$

in the two-dimensional Ricker competition model (31) imply its global stability provided that $1<r_{1}, r_{2}<2$, and the image of a critical curves [30] do not intersect. Later, Ryals and Sacker [31] improved the result by reducing the infinite number of topological conditions on the critical curves to a finite number.

We should mention that Smith [32] uses monotonicity to prove the global stability of the fixed points of the system

$$
\begin{aligned}
& u_{n+1}=u_{n} \exp \left(r\left(1-u_{n}-B v_{n}\right)\right) \\
& v_{n+1}=v_{n} \exp \left(s\left(1-C u_{n}-v_{n}\right)\right),
\end{aligned}
$$

when $r, s \leq 1$, in which case the invariant set is $\left[0, r^{-1}\right] \times$ $\left[0, s^{-1}\right]$. Notice that, by the changes of variables $r u=x$ and $s v=y$, system $(70)$ is equivalent to

$$
\begin{aligned}
& x_{n+1}=x_{n} \exp \left(r-x_{n}-\frac{B r}{s} y_{n}\right) \\
& y_{n+1}=y_{n} \exp \left(s-y_{n}-\frac{C s}{r} x_{n}\right) .
\end{aligned}
$$

Clearly, $r_{1}=r, r_{2}=s, c_{12}=\mathrm{Br} / \mathrm{s}$, and $c_{21}=\mathrm{Cs} / \mathrm{r}$. Therefore, Smith's global results cover the local analysis when the carrying capacities are taken in the unit interval and, in this case, global stability is obtained when $r_{i} \leq 1$, for $i=1,2$, such that $r_{i} \in S_{1}$ (Figure 3 ).

It remains as an open problem to show the global stability in the mixing cases, that is, when $r_{1} \leq 1$ and $1<r_{2} \leq 2$, or $r_{2} \leq 1$ and $1<r_{1} \leq 2$, and also in the region between $r_{1}=2$ or $r_{2}=2$ and the branch of hyperbola $\gamma_{1}$ as is depicted in Figure 3 , as part of the region $S_{1}$. This is precisely part of the conjecture raised by Elaydi and Luís [33].

We should mention that the study of global stability can also be extended to the other nontrivial fixed points (by trivial fixed point we mean the origin) of system (31), as well.

Now, motivated by the precedent cases in lower dimension, we have the following conjecture concerning the nontrivial fixed points of the three-dimensional Ricker competition model.

Conjecture 15. Consider the Ricker competition model (28) where the growth rates $r_{i}$ are positive and the competition parameters are taken as $c_{i i}=1$ and $0<c_{j i}<1$, for $i \neq j$ and $i, j=1,2,3$. Let $\mathbf{x}^{*}$ be a nontrivial fixed point of the map $F$ defined in (29) and assume that we have

$$
\begin{aligned}
& -1-\sum_{i=1}^{3} M_{i i}\left(J F\left(\mathbf{x}^{*}\right)\right)<\operatorname{tr}\left(J F\left(\mathbf{x}^{*}\right)\right)+\operatorname{det}\left(J F\left(\mathbf{x}^{*}\right)\right) \\
& \leq 1+\sum_{i=1}^{3} M_{i i}\left(J F\left(\mathbf{x}^{*}\right)\right)
\end{aligned}
$$




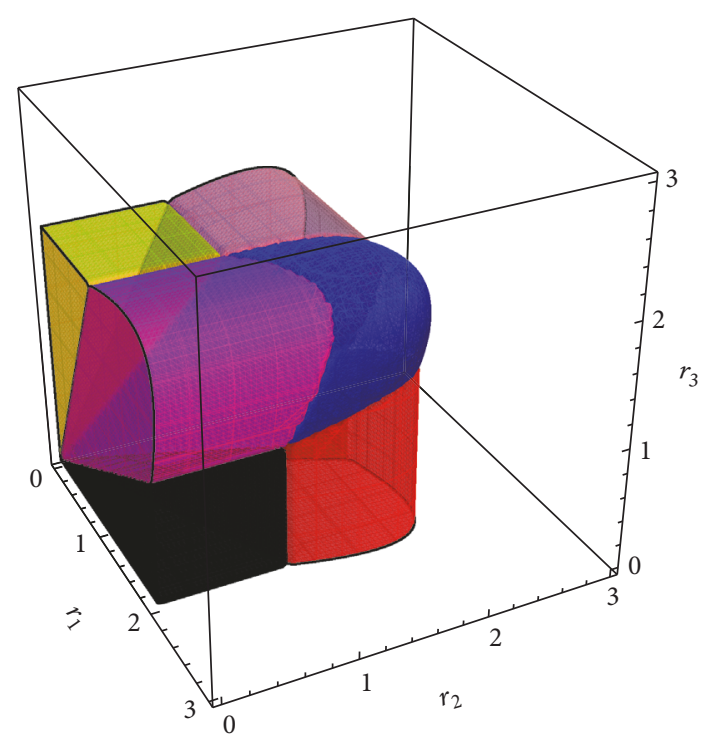

FIGURE 7: Stability regions, in the parameter space $\mathrm{Or} r_{1} r_{2} r_{3}$, of the fixed points of the three-dimensional Ricker competition model when $c_{i i}=1$ and $c_{j i}=0.5$, for $i \neq j$ and $i, j=1,2,3$.

$$
\begin{aligned}
& \left|\sum_{i=1}^{3} M_{i i}\left(J F\left(\mathbf{x}^{*}\right)\right)-\operatorname{tr}\left(J F\left(\mathbf{x}^{*}\right)\right) \operatorname{det}\left(J F\left(\mathbf{x}^{*}\right)\right)\right| \\
& \quad<1-\operatorname{det}^{2}\left(J F\left(\mathbf{x}^{*}\right)\right),
\end{aligned}
$$

where $\operatorname{tr}\left(J F\left(\mathbf{x}^{*}\right)\right), \operatorname{det}\left(J F\left(\mathbf{x}^{*}\right)\right)$, and $\sum_{i=1}^{3} M_{i i}\left(J F\left(\mathbf{x}^{*}\right)\right)$ are the trace, the determinant, and the sum of the principal minors of the Jacobian matrix evaluated at the fixed point $\mathbf{x}^{*}$, respectively.

Then, the fixed point $\mathbf{x}^{*}$ is globally asymptotically stable with respect to the interior of the first octant.

Notice that, for each one of the nontrivial fixed points $\mathbf{x}^{*}$ mentioned in the conjecture (three fixed points on the axes $E_{1}, E_{2}$, and $E_{3}$, three fixed points on the planes $E_{12}$, $E_{13}$, and $E_{23}$, and the positive fixed point $\left.E^{*}\right)$, the local stability conditions (72) correspond to those developed in the previous section for the respective fixed point. Moreover, geometrically it corresponds to each one of the seven regions depicted in Figure 7 as mentioned before.

Before ending this section, we should mention that in a forthcoming paper, Balreira et al. [34] use a new notion of monotonicity in higher dimensional maps and show that Conjecture 15 is true in the case of the positive fixed point $E^{*}$, when $r_{i}<1$ for $i=1,2,3$. The result was established since the three-dimensional Ricker competition model is monotone (more specifically, normally monotone) if conditions (72) are valid such that $r_{i}<1$ for $i=1,2,3$. This is precisely a subset of the set depicted in Figure 6.

Finally, if one considers the remaining region in the parameter space, the map is not monotone. Since it is possible to show global stability, in the planar model, for certain cases (Balreira et al. [28] and Ryals and Sacker [31]), using the Singularity Theory of Whitney [29], we believe that an extension of the ideas may be useful in proving Conjecture 15. Unfortunately, such theory of singularity does not exist for three-dimensional systems. It either is necessary to develop such theory of singularity in three-dimensional models to show Conjecture 15 or may use another different tool such as Lyapunov method (cf. Theorem 4.2 in Elaydi [20, page 205]).

\section{Conclusion}

In this paper we illustrate how to apply the centre manifold theorem to three-dimensional discrete dynamical systems. We recall the necessary and sufficient conditions of local stability of three-dimensional systems and give, in depth, the tools to study the stability of nonhyperbolic fixed points in the terminology of difference equations.

As illustrative example, we study the Ricker competition model of three species. This nonlinear model has seven nontrivial fixed points. Each fixed point has a region, in the parameter space, where it is locally asymptotically stable. When one determines these regions, the result is the seven regions depicted in Figure 7.

In addition, we state a conjecture in the global stability of each nontrivial fixed point of the Ricker competition model, in the respective region of local stability.

\section{Conflicts of Interest}

The authors declare that there are no conflicts of interest regarding the publication of this paper.

\section{Acknowledgments}

This work was partially supported by FCT/Portugal through UID/MAT/04459/2013.

\section{References}

[1] G. Osipenko, "Center manifolds," Encyclopedia of Complexity and Systems Science, pp. 936-951, 2009.

[2] A. M. Lyapunov, The general problem of the stability of motion (in Russian) [Phd. Dissertation], University of Kharkov 1892, (English translation version: (1) Stability of Motion (Academic Press, New York, 1966).(2) The General Problem of the Stability of Motion (Taylor and Francis, London, 1992)).

[3] V. A. Pliss, "A reduction principle in the theory of stability of motion," Izvestiya Akademii Nauk SSSR. Seriya Matematicheskaya, vol. 28, pp. 1297-1324, 1964.

[4] A. Kelley, "The stable, center-stable, center, center-unstable, unstable manifolds," Journal of Differential Equations, vol. 3, pp. 546-570, 1967.

[5] G. Iooss, Bifurcation of maps and applications, vol. 36 of NorthHolland Mathematics Studies, North-Holland Publishing Co., Amsterdam-New York, 1979.

[6] J. Carr, Applications of Centre Manifold Theory, Springer, New York, NY, USA, 1981.

[7] J. Guckenheimer and P. Holmes, Nonlinear Oscillations, Dynamical Systems, and Bifurcation of Vector Fields, Springer, New York, NY, USA, 1983. 
[8] Y. A. Kuznetsov, Elements of Applied Bifurcation Theory, vol. 112 of Applied Mathematical Sciences, Springer, New York, NY, USA, 3rd edition, 2004.

[9] S. N. Elaydi, Discrete Chaos: with Applications in Science and Engineering, Chapman \& Hall, Boca Raton, Fla, USA, 2nd edition, 2008.

[10] S. Wiggis, Introduction to Applied Nonlinear Dynamical Systems, vol. 2, Springer-Verlag, New York, 2003.

[11] W. Zhang, "One-dimensional dynamical economic systems," in Discrete Dynamical Systems, Bifurcations and Chaos in Economics, vol. 204 of Mathematics in Science and Engineering, pp. 79-134, Elsevier, 2006.

[12] J. Dektešević, M. R. Kulenović, and E. Pilav, "Asymptotic approximation of a stable and unstable manifolds of a twodimensional quadratic map," Journal of Computational Analysis and Applications, vol. 21, no. 1, pp. 35-51, 2016.

[13] M. Guzowska, R. Luis, and S. Elaydi, "Bifurcation and invariant manifolds of the logistic competition model," Journal of Difference Equations and Applications, vol. 17, no. 12, pp. 1851-1872, 2011.

[14] N. Karydas and J. Schinas, "The center manifold theorem for a discrete system," Applicable Analysis. An International Journal, vol. 44, no. 3-4, pp. 267-284, 1992.

[15] M. R. Kulenović and O. Merino, "Invariant manifolds for competitive discrete systems in the plane," International Journal of Bifurcation and Chaos in Applied Sciences and Engineering, vol. 20, no. 8, pp. 2471-2486, 2010.

[16] R. Luis, S. Elaydi, and H. Oliveira, "Stability of a Ricker-type competition model and the competitive exclusion principle," Journal of Biological Dynamics, vol. 5, no. 6, pp. 636-660, 2011.

[17] N. Psarros, G. Papaschinopoulos, and C. J. Schinas, "Semistability of two systems of difference equations using centre manifold theory," Mathematical Methods in the Applied Sciences, vol. 39, no. 18, pp. 5216-5222, 2016.

[18] T. Alligood, T. D. Sauer, and J. A. Yorke, Chaos: An Introduction to Dynamical Systems, Springer, New York, NY, USA, 1996.

[19] R. L. Devaney, An Introduction To Chaotic Dynamical Systems, Westview Press, Boulder, Colo, USA, 2nd edition, 2003.

[20] S. Elaydi, An Introduction to Difference Equations, Springer, New York, NY, USA, 3rd edition, 2005.

[21] B. P. Brooks, "Linear stability conditions for a first-order threedimensional discrete dynamic," Applied Mathematics Letters. An International Journal of Rapid Publication, vol. 17, no. 4, pp. 463466, 2004.

[22] J. Marsden and M. McCracken, The Hopf Bifurcation and Its Applications, Springer, New York, NY, USA, 1976.

[23] E. Ricker, "Handbook of Computation for Biological Statistics of Fish Populations," Bulletin 119 of the Fisheries Resource, 1958.

[24] J. M. Cushing, An Introduction to Structured Population Dynamics, vol. 71, The Society for Industrial and Applied Mathematics (SIAM), Philadelphia, Pa, USA, 1998.

[25] A. N. Sharkovsky, "Coexistence of cycles of a continuous map of the line into itself," Ukrainian Mathematical Journal, vol. 16, pp. 61-71, 1964 (Russian).

[26] A. N. Sharkovski, "Coexistence of cycles of a continuous map of the line into itself (translation to English by J. Tolosa)," International Journal of Bifurcation and Chaos, vol. 5, no. 5, pp. 1263-1273, 1995.

[27] W. A. Coppel, “The solution of equations by iteration," Mathematical Proceedings of the Cambridge Philosophical Society, vol. 51, no. 1, pp. 41-43, 1955.
[28] E. C. Balreira, S. Elaydi, and R. Luís, "Local stability implies global stability for the planar Ricker competition model," Discrete and Continuous Dynamical Systems. Series B. A Journal Bridging Mathematics and Sciences, vol. 19, no. 2, pp. 323-351, 2014.

[29] H. Whitney, "On singularities of mappings of euclidean spaces. Mappings of the plane into the plane," Annals of Mathematics, vol. 62, no. 3, pp. 374-410, 1955.

[30] A. Barugola, C. Mira, L. Gardini, and J. Cathala, Chaotic Dynamics in Two-Dimensional Noninvertible Maps, Nonlinear Sciences Series A, World Scientific, Singapore, 1996.

[31] B. Ryals and R. J. Sacker, "Global stability in the 2D Ricker equation," Journal of Difference Equations and Applications, vol. 21, no. 11, pp. 1068-1081, 2015.

[32] H. L. Smith, "Planar competitive and cooperative difference equations," Journal of Difference Equations and Applications, vol. 3, no. 5-6, pp. 335-357, 1998.

[33] S. Elaydi and R. Luís, "Open problems in some competition models," Journal of Difference Equations and Applications, vol. 17, no. 12, pp. 1873-1877, 2011.

[34] E. C. Balreira, S. Elaydi, and R. Luís, "Global stability of higher dimensional monotone maps," Journal of Difference Equations and Applications, In press. 


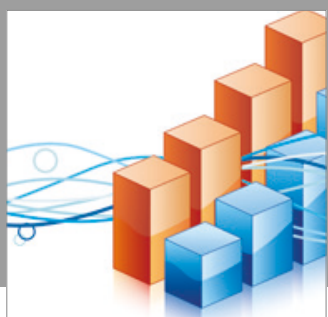

Advances in

Operations Research

vatersals

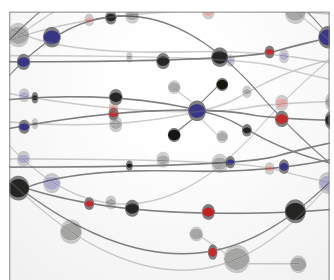

\section{The Scientific} World Journal
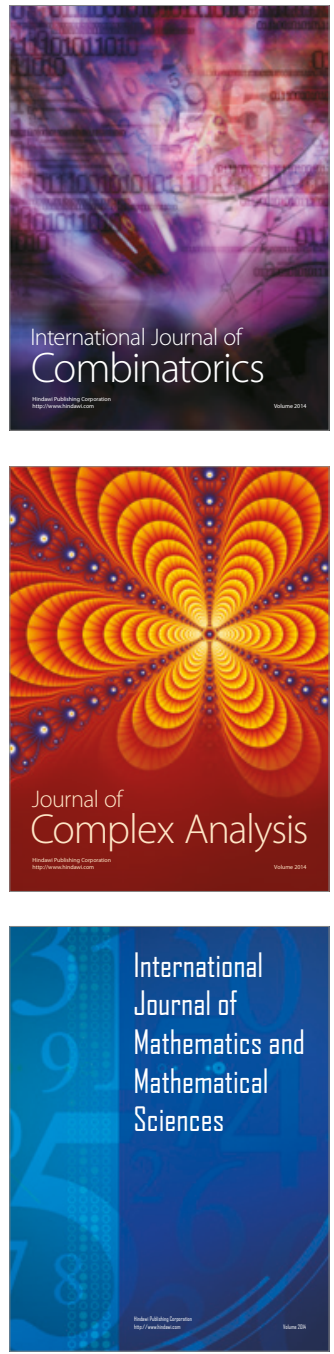
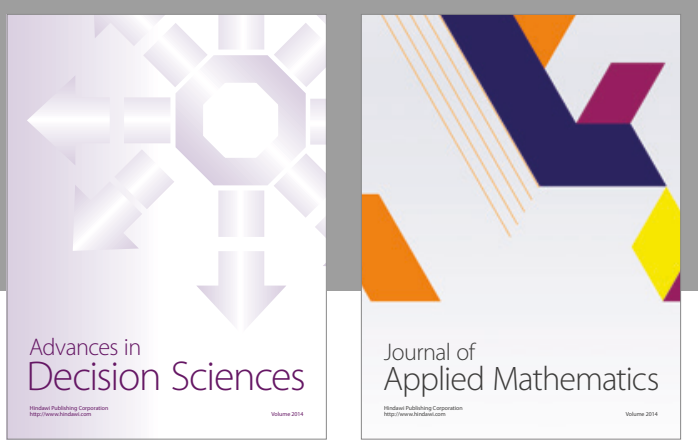

Algebra

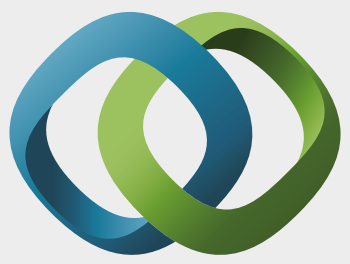

\section{Hindawi}

Submit your manuscripts at

https://www.hindawi.com
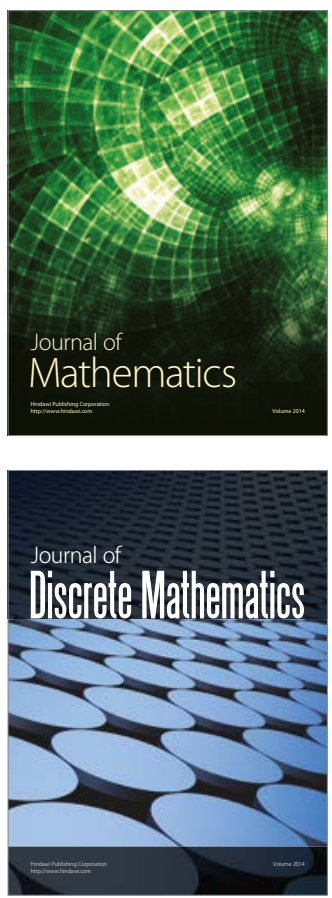

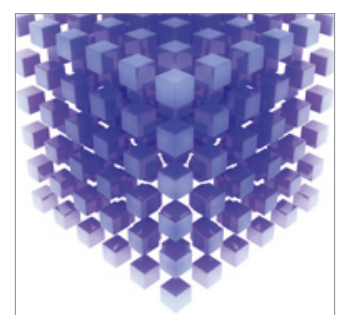

Mathematical Problems in Engineering
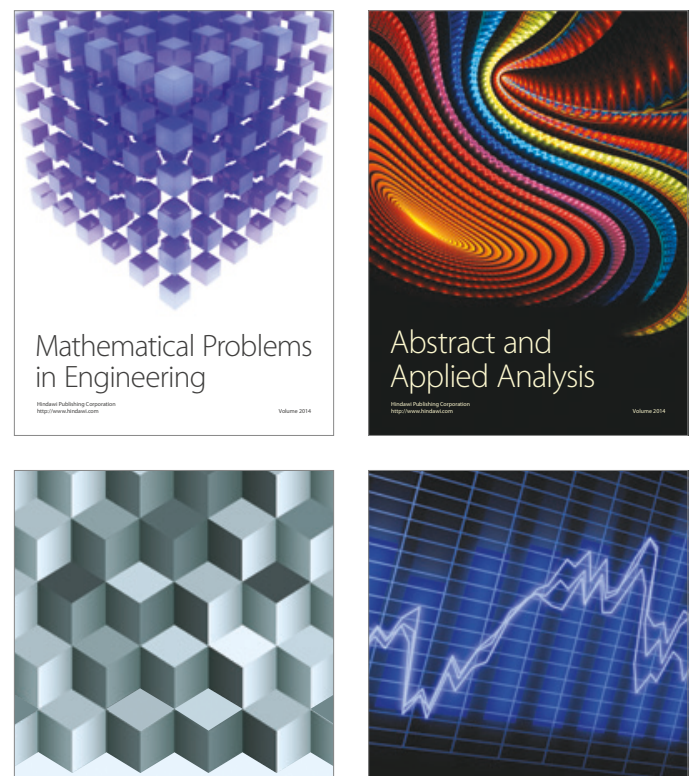

Journal of

Function Spaces

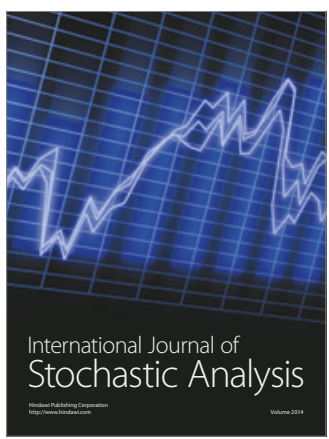

Probability and Statistics
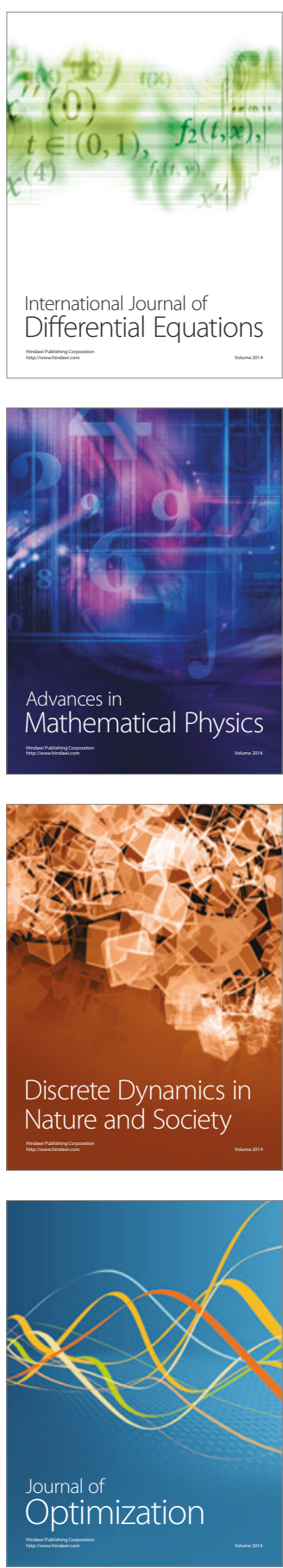